\title{
A Theoretical Investigation Based on the Rough Approximations of Hypergraphs
}

\author{
Musavarah Sarwar (i) \\ Department of Mathematics, Government College Women University, Sialkot, Pakistan \\ Correspondence should be addressed to Musavarah Sarwar; musavarah656@gmail.com
}

Received 11 December 2021; Accepted 5 January 2022; Published 7 March 2022

Academic Editor: Gohar Ali

Copyright (C) 2022 Musavarah Sarwar. This is an open access article distributed under the Creative Commons Attribution License, which permits unrestricted use, distribution, and reproduction in any medium, provided the original work is properly cited.

Rough sets are a key tool to model uncertainty and vagueness using upper and lower approximations without predefined functions and additional suppositions. Rough graphs cannot be studied more effectively when the inexact and approximate relations among more than two objects are to be discussed. In this research paper, the notion of a rough set is applied to hypergraphs to introduce the novel concept of rough hypergraphs based on rough relations. The notions of isomorphism, conformality, linearity, duality, associativity, commutativity, distributivity, Helly property, and intersecting families are illustrated in rough hypergraphs. The formulae of 2-section, L2-section, covering, coloring, rank, and antirank are established for certain types of rough hypergraphs. The relations among certain types of products of rough hypergraphs are studied in detail.

\section{Introduction}

In graphical networks, usually pairwise relations are discussed missing some information that more than two objects may satisfy common characteristics. Hypergraphs introduced by Berge [1] as a generalization of graphs tackle the difficulty to study relations and common characteristics of any set of objects. A lot of work has been done on hypergraphs due to their applications in various domains of biological and computer sciences including properties and algorithms of the Cartesian product of hypergraphs [2], the direct product of hypergraphs [3], and hamiltonicity of certain products of hypergraphs [4]. Hammack et al. [5] studied distance measures, isometries, factorization, chromaticity, and various other properties of graph products which is a strong framework to generalize all the results for hypergraphs.

Hypergraphs are the key tool to study real-world problems in a more generalized and efficient way as compared to graphs and their extensions but are unable to study uncertainty and vagueness occurring in data and incomplete information. Kaufmann [6] extended the concept of hypergraphs to fuzzy hypergraphs by applying fuzzy sets [7] on hypergraphs. Lee-Kwang and Lee [8] proved that there are some flaws in Kaufmann's definition of a fuzzy hypergraph and redefined that concept. Radhamani and Radhika [9] added fuzzy relations in fuzzy hypergraphs and initiated the edited concept of fuzzy hypergraphs with certain isomorphism properties. Researchers are continuously working on the properties of fuzzy hypergraphs and their extensions including Hebbian structures of fuzzy hypergraphs [10], fuzzy coloring in fuzzy hypergraphs [11], transversals of fuzzy hypergraphs [12], certain properties of fuzzy hypergraphs [13], intuitionistic fuzzy hypergraphs [14, 15], bipolar fuzzy hypergraphs [16], various extensions of hypergraphs to deal with uncertainty [17], $m$-polar fuzzy hypergraphs $[18,19]$, and bipolar fuzzy soft hypergraphs [20].

All the existing approaches of hypergraphs based on fuzzy sets and their extensions can be applied using membership functions and parameterization tools. But, in some situations, when we have no additional information, membership functions, or parametric properties, the existing models based on hypergraphs are difficult to apply. Rough sets, introduced by Pawlak [21], are a key tool to handle such situations and study uncertain information without membership functions using upper and lower approximations. Rough sets are becoming a wide domain of research to study hybrid models based on graphs, relations, 
decision making problems, and fuzzy models, for instance, rough relations [22], rough graphs [23], rough fuzzy digraphs [24], fuzzy rough graphs [25], hybrid models based on rough sets, soft sets, and graphs [26], soft rough sets and rough soft sets [27], soft rough fuzzy sets [28], fuzzy sets combined with rough sets [29], rough set approximations for big data systems [30], hypergroups based on upper and lower approximations [31], modeling similarities in rough set theories [32], properties of certain types of rough relations [33], image classification based on rough sets [34], fuzzy rough feature selection based on graphs [35], risk minimization based in rough sets [36], fuzzy FCA (formal concept analysis) based on rough sets [37], applications of rough sets to graphs [38], l2-hypergraphs [39], vertex rough graphs [40], FCA based on hypergraphs and rough sets [41], weak chromatic number of random hypergraphs [42], properties of totally balanced hypergraphs [43], Boolean operators based on rough sets [44], boundary optimization for rough sets [45] and connection between hypergroups, rough sets and hypergraphs [46], domination based rough sets [47], relationships between rough sets and topologies [48], planarity of product graphs in bipolar fuzzy environment [49], bipolar soft sets based on rough multipolar fuzzy approximations [50], and bipolar soft sets under rough Pythagorean fuzzy environment [51].

1.1. Motivation and Contribution. The motives of the present study are summarized as follows:

(1) Graph theory has a wide range of applications in different domains to study pairwise relations among objects. But, in graphical models, usually certain information is ignored that two or more objects may satisfy common properties or characteristics. Hypergraph theory as a generalization of graph theory tackles this difficulty to study common characteristics of any collection of objects in a more efficient way.

(2) In a hypergraph, binary values 0 and 1 are used to identify whether certain objects satisfy a common characteristic or not. Hypergraphs cannot study uncertain properties or partial belongingness of objects and their relations. Fuzzy hypergraphs and their extensions have been applied successfully to deal with uncertain information in hypergraphical models. But, all these existing approaches are based on additional suppositions and membership functions to compute the vagueness of objects. Rough sets are a power tool to discuss uncertainty using upper and lower approximations without any additional assumptions and predefined functions. Rough hypergraphs as an extension of hypergraphs can study incomplete information in hypergraphical models using given information, that is, no need for additional assumptions, which is the main focus of the present study.

(3) Various hypergraphical structures have applications for map learning, link prediction, information entropy, etc. Hypergraphs are usually used to represent relations among social objects. Rough hypergraphs can be used to cope with uncertain relations among objects in social networks without suppositions of arbitrary membership values and functions unlike fuzzy hypergraphs and their extensions. Rough hypergraphs can be used in decision analysis for the grouping of different teams, storage of incompatible and flammable substances, and route-finding problems using distance measures. follows:

The main contribution of this research paper is as

(1) This study proposes the novel concept of rough hypergraph using rough relations. A rough hypergraph is constructed on a set using equivalence relations.

(2) The properties of isomorphism, conformality, linearity, duality, associativity, commutativity, distributivity, Helly property, and intersecting families in rough hypergraphs are studied in detail.

(3) Certain operations on rough hypergraphs are discussed. The formulae for 2-section, L2-section, covering, coloring, rank, and antirank are established using approximation techniques.

1.2. Framework of the Paper. This paper is organized as follows:

(1) Section 1 is based on the literature review and motives of the given study

(2) Section 2 contains basic ideas, definitions, and terminologies from already existing articles that are used in the paper

(3) Section 3 is the main focus of this research paper which contains novel concepts of certain types of rough hypergraphs and their interesting properties

\section{Preliminaries}

A hypergraph [1] on a nonempty set $Q$ is written as a pair $H=(Q, D)$, where $D=\left\{D_{1}, D_{2}, \ldots, D_{r}\right\}$ is a family of nonempty subsets of $Q$ such that $\cup_{k=1}^{r} D_{k}=Q$.

Definition 1 (see [21]). An approximation space on $T$ is a pair $(Q, \varphi)$, where $\varphi$ is defined as an equivalence (EQ) relation on $T$. For any subset $A \subseteq Q$, the upper approximation $\bar{\varphi} A$ and lower approximation $\underline{\varphi} A$ of $A$ in $(Q, \varphi)$ are defined as

$$
\begin{aligned}
\bar{\varphi} A & =\left\{d \in Q \mid[d]_{\varphi} \cap A \neq \varnothing\right\}, \\
\underline{\varphi} A & =\left\{d \in Q \mid[d]_{\varphi} \subseteq A\right\} .
\end{aligned}
$$

Here, $[d]_{\varphi}=\{g \in Q \mid(d, g) \in \varphi\}$ is known as EQ class of $d \in T$, and the pair $(\varphi A, \bar{\varphi} A)$ is called a rough set.

Definition 2 (see [23]). A rough digraph on a nonvoid set $Q$ is a 3-tuple $\mathscr{G}=(Q, \varphi A, \psi D)$ such that 
(1) $\varphi$ is an EQ relation on the vertex set $Q$

(2) For $A \subseteq Q, \varphi A=(\varphi A, \bar{\varphi} A)$ is a rough set on $Q$

(3) $\psi D$ is an EQ relation on any $M \subseteq Q \times Q$

(4) For $D \subseteq A \times A, \psi D=(\psi D, \bar{\psi} D)$ is a rough relation on $\varphi A=(\varphi A, \bar{\varphi} A)$

The rough digraph is also represented by the pair $\mathcal{U}=(\underline{\mathcal{U}}, \overline{\mathcal{U}})$, where $\underline{\mathcal{U}}=(\varphi A, \psi D)$ and $\overline{\mathcal{U}}=(\bar{\varphi} A, \bar{\psi} D)$ are digraphs. If $D$ is an irreflexive and symmetric relation, $\underline{\mathcal{U}}$ and $\overline{\mathcal{U}}$ are simple graphs and $\mathcal{U}$ is a rough graph on $Q$.

\section{Rough Hypergraphs}

In this section, the notion of a rough hypergraph is introduced with certain interesting properties of isomorphism, linearity, duality, and rough line graphs. We have discussed the 2-section, L2-section, rank, antirank, covering, and coloring of certain operations of rough hypergraphs.

Definition 3. Let $\varphi$ be an EQ relation on $Q$ and for $A \subseteq Q$; let $(\varphi A, \bar{\varphi} A)$ be a rough set on $Q$. Let $\psi$ be an EQ relation on $\overline{\mathscr{M}} \subseteq \mathscr{P}(Q) \backslash\{\varnothing\}$, the power set of $Q$, such that for each $d_{1}, g_{1} \in U_{1}$, there exist $d_{2}, g_{2} \in U_{2}$ iff $\left(U_{1}, U_{2}\right) \in \psi$.

Let $D \subseteq \mathscr{P}(A) \backslash\{\varnothing\}$ be a family of nonempty subsets of $A$; then the upper and lower approximations $\bar{\psi} D$ and $\psi D$ are defined as

$$
\begin{aligned}
& \bar{\psi} D=\left\{V \in \mathscr{M} \mid[V]_{\psi} \cap D \neq \varnothing\right\}, \\
& \underline{\psi} D=\left\{V \in \mathscr{M} \mid[V]_{\psi} \subseteq D\right\} .
\end{aligned}
$$

The pair $(\psi D, \bar{\psi} D)$ is known as a rough relation on $Q$. If $\psi D \subseteq \mathscr{P}(\varphi A)$, the power set of $\varphi A$, then $(\psi D, \bar{\psi} D)$ is a rough relation on $(\varphi A, \bar{\varphi} A)$.

Definition 4. A rough hypergraph on a nonempty set $Q$ is a triplet $(Q, \varphi A, \psi D)$ such that

(1) $\varphi$ is an EQ relation on vertex set $Q$

(2) For $A \subseteq Q,(\varphi A, \bar{\varphi} A)$ is a rough set on $Q$

(3) $\psi$ is an EQ relation on $\mathscr{M} \subseteq \mathscr{P}(Q) \backslash\{\varnothing\}$, that is, the family of nonempty subsets of $Q$

(4) For $D \subseteq \mathscr{P}(A) \backslash\{\varnothing\},(\psi D, \bar{\psi} D)$ is a rough relation on $(\varphi A, \bar{\varphi} A)$; that is, $\psi D \overline{D \subseteq} \mathscr{P}(\varphi A)$

The rough hypergraph on $Q$ is also denoted by the pair $\mathscr{R}=(\underline{\mathscr{R}}, \overline{\mathscr{R}})$, where $\underline{\mathscr{R}}=(\varphi A, \psi D)$ and $\overline{\mathscr{R}}=(\bar{\varphi} A, \bar{\psi} D)$ are hypergraphs.

Example 1. Let $\varphi$ be an EQ relation on $Q=\{o, d, t, g, y, h\}$ as given in Figure 1. Let $A=\{o, t, g, d, h\}$, then $\varphi A=\{o, h, d, t\}$, and $\bar{\varphi} A=\{o, d, t, g, y, h\}$. Let $\psi$ be an EQ relation on $\mathscr{M} \subseteq \mathscr{P}(Q) \backslash\{\varnothing\}$ as shown in Figure 2 .

Let $D=\{\{o, g, h\},\{o, t, d, y\},\{o, t, d, g\}\}$, then clearly $\psi D=\{\{\{o, t, d, y\},\{o, t, d, g\}\}, \quad$ and $\bar{\psi} \quad \mathrm{D}=\{\{o, g, h\}$, $\{o, y, h\},\{o, g, y, h\},\{o, t, d, g\},\{o, t, d, y\}\}$. The rough hypergraph on $Q$ is shown in Figure 3.

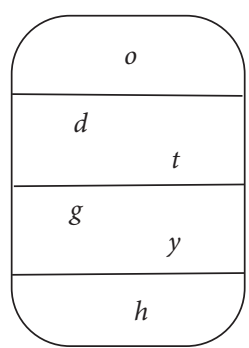

FIgURE 1: Approximation space $(Q, \varphi)$.

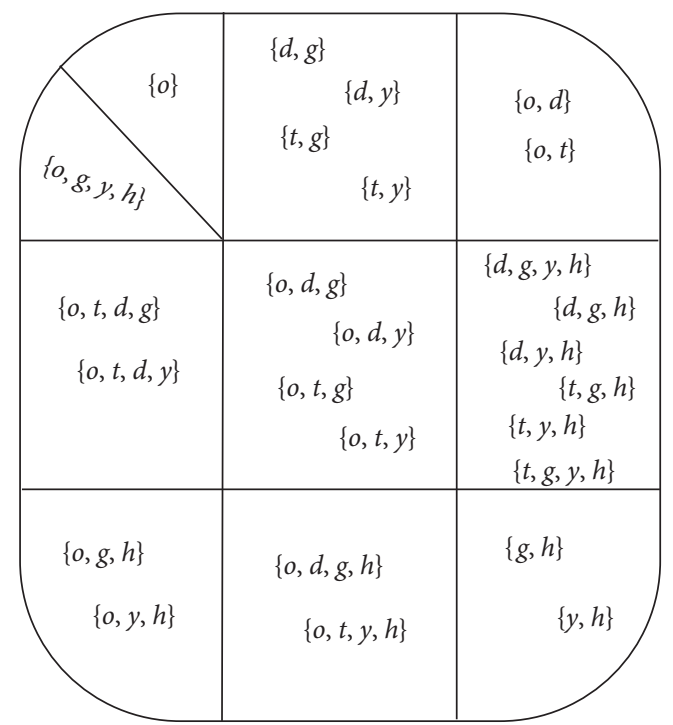

Figure 2: Approximation space $(\mathscr{M}, \psi)$.
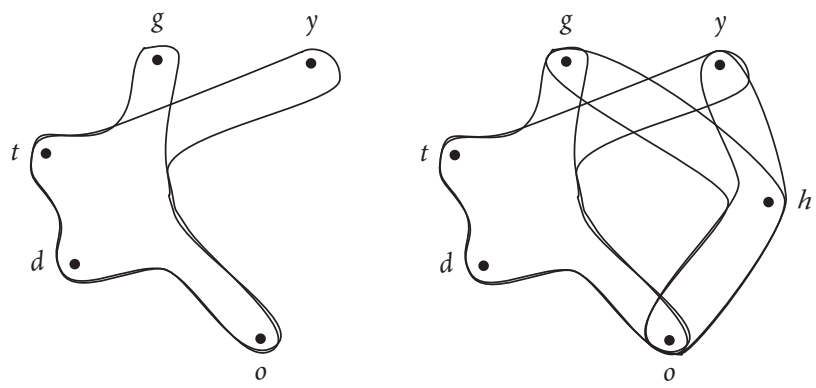

Figure 3: Rough hypergraph $((\varphi A, \psi D),(\bar{\varphi} A, \bar{\psi} D))$.

Definition 5. The degree of a vertex $k$ in a rough hypergraph $\mathscr{R}=(\underline{R}, \overline{\mathscr{R}})$ is the sum of degrees of vertex $k$ in both hypergraphs $\underline{\mathscr{R}}=(\psi A, \psi D)$ and $\overline{\mathscr{R}}=(\bar{\psi} A, \bar{\psi} D)$. It is denoted as $\operatorname{deg}(\bar{k})=(1 / 2)(\operatorname{deg}(k)+\overline{\operatorname{deg}}(k))$, where $\operatorname{deg}(k)$ and $\overline{\operatorname{deg}}(k)$ denote the number of hyperedges incident to $k$ in $\underline{\mathscr{R}}(k)$ and $\overline{\mathscr{R}}(k)$, respectively.

The maximum degree of a rough hypergraph is denoted by $\Delta(\mathscr{R})$ and computed as the sum of maximum degrees in $\underline{\mathscr{R}}$ and $\overline{\mathscr{R}}$, respectively; that is, $\Delta(\mathscr{R})=(1 / 2)(\Delta(\underline{\mathscr{R}})+$ $\Delta(\overline{\mathscr{R}}))=(1 / 2)\left(\max _{k \in P} \operatorname{deg}(k)+\max _{k \in P} \overline{\operatorname{deg}}(k)\right)$. 
The minimum degree of a rough hypergraph is denoted by $\delta(\mathscr{R})$ and computed as the sum of minimum degrees in $\underline{\mathscr{R}}$ and $\overline{\mathscr{R}}$, respectively; that is, $\delta(\mathscr{R})=(1 / 2)$ $(\delta(\underline{\mathscr{R}})+\delta(\overline{\mathscr{R}}))=(1 / 2)\left(\max _{k \in P} \operatorname{deg}(k)+\min _{k \in P} \overline{\operatorname{deg}}(k)\right)$.

Definition 6. The rank of a rough hypergraph is denoted by $r(\mathscr{R})$ and defined as the sum of ranks of $\underline{\mathscr{R}}$ and $\overline{\mathscr{R}}$; that is, $r(\mathscr{R})=(1 / 2)(r(\underline{\mathscr{R}})+r(\overline{\mathscr{R}}))=(1 / 2)\left(\max _{E \in \psi}|E|+\right.$ $\left.\max _{E \in \bar{\psi} D}|E|\right)$.

The antirank of a rough hypergraph is denoted by $s(\mathscr{R})$ and defined as the sum of antiranks of $\underline{\mathscr{R}}$ and $\overline{\mathscr{R}}$; that is, $s \mathscr{R})=s(\underline{\mathscr{R}})+s(\overline{\mathscr{R}})=(1 / 2)\left(\max _{k \in P} \overline{\min }_{E \in \psi D}|E|+\right.$ $\left.\min _{E \in \bar{\psi} D}|E|\right)$.

A rough hypergraph is called a uniform rough hypergraph if $r(\mathscr{R})=s(\mathscr{R})$. A uniform rough hypergraph is known as a $p$ - uniform rough hypergraph if $r(\underline{\mathscr{R}})=r(\overline{\mathscr{R}})=p$.

Note that a rough hypergraph for which $r(\underline{\mathscr{R}}) \leq 2$ and $r(\overline{\mathscr{R}}) \leq 2$ is a rough graph. A 2 -uniform rough hypergraph is a rough graph.

Definition 7. A rough hypergraph $\mathscr{R}=((\varphi A$, $\psi D),(\bar{\varphi} A, \bar{\psi} D))$ is called a partial rough hypergraph of a rough hypergraph $\mathcal{S}=((\varphi B, \psi F),(\bar{\varphi} B, \bar{\psi} F))$ if $\varphi A \subseteq \varphi B$, $\psi D \subseteq \psi F, \bar{\varphi} A \subseteq \bar{\varphi} B$, and $\bar{\psi} D \subseteq \bar{\psi} F$. It is written as $\mathscr{R} \subseteq \overline{\mathcal{S}}$.

A partial rough hypergraph $\mathscr{R}=((\varphi A, \psi D),(\bar{\varphi} A, \bar{\psi} D))$ is called induced if $\psi F$ and $\bar{\psi} F$ contain all hyperedges of $\mathcal{S}$ which has vertices from $\underline{\varphi} B$ and $\bar{\varphi} B$, respectively.

Definition 8. A rough hyperpath of length $q$ between vertices $g$ and $k$, denoted by $k_{o}--k_{q}$, in a rough hypergraph $\mathscr{R}=((\varphi A, \psi D),(\bar{\varphi} A, \bar{\psi} D))$ is a sequence of distinct vertices and hyperedges $k_{o}, D_{1}, k_{1}, D_{2}, k_{2}, D_{3}, \ldots, D_{q-1}, k_{q-1}, D_{q}, k_{q}$ in both $\underline{\mathscr{R}}=(\varphi A, \psi D)$ and $\overline{\mathscr{R}}=(\bar{\varphi} A, \bar{\psi} D)$. If $k_{o}=k_{q}$, then the rough hyperpath is known as a rough hypercycle.

Definition 9. The distance $d_{\mathscr{R}}(k, g)$ between any two vertices $k$ and $g$ of a rough hypergraph $\mathscr{R}$ is defined as the sum of lengths of shortest hyperpaths connecting $k$ and $g$ in both $\underline{\mathscr{R}}$ and $\overline{\mathscr{R}}$; that is, $d_{\mathscr{R}}(k, g)=(1 / 2)\left(d_{\mathscr{R}}(k, g)+d_{\overline{\mathscr{R}}}(k, g)\right)$.

Definition 10 . Let $\mathscr{R}_{1}$ and $\mathscr{R}_{2}$ be two rough hypergraphs on $Q_{1}$ and $Q_{2}$, respectively. A homomorphism from $\mathscr{R}_{1}$ into $\mathscr{R}_{2}$ is a mapping $f: Q_{1} \longrightarrow Q_{2}$ if there exist homomorphisms $\underline{f}: Q_{1} \longrightarrow Q_{2}$ and $\bar{f}: Q_{1} \longrightarrow Q_{2}$; that is,

(1) If $\bar{f}(D)$ is a hyperedge in $\overline{\mathscr{R}_{2}}$, then $D$ is a hyperedge in $\overline{\mathscr{R}_{1}}$

(2) If $f(D)$ is a hyperedge in $\mathscr{R}_{2}$, then $D$ is a hyperedge in $\mathscr{R}_{1}$

A homomorphism which is a one-to-one correspondence between $Q_{1}$ and $Q_{2}$ is called an isomorphism. In this case, we say that the rough hypergraphs $\mathscr{R}_{1}$ and $\mathscr{R}_{2}$ are isomorphic to each other and write as $\mathscr{R}_{1} \cong \mathscr{R}_{2}$.
Definition 11. The 2-section $[\mathscr{R}]_{2}=\left([\mathscr{R}]_{2},[\overline{\mathscr{R}}]_{2}\right)=$ $\left(\left(\varphi A,[\psi D]_{2}\right),\left(\bar{\varphi} A,[\bar{\psi} D]_{2}\right)\right)$ of a rough hypergraph $\mathscr{R}=(\underline{\mathscr{R}}, \overline{\mathscr{R}})$ is a rough graph with the same vertex set as in $\mathscr{R}$ and two vertices are adjacent in $[\underline{\mathscr{R}}]_{2}$ and $[\overline{\mathscr{R}}]_{2}$ if they belong to the same hyperedge in $\mathscr{R}$ and $\overline{\mathscr{R}}$, respectively.

The L2-section $[\mathscr{R}]_{L 2}=\left([\underline{\mathscr{R}}]_{L 2},[\overline{\mathscr{R}}]_{\bar{L} 2}\right)=\left(\left(\varphi A,[\psi D]_{2}\right.\right.$, $\left.\underline{L}),\left(\bar{\varphi} A,[\bar{\psi} D]_{2}, \bar{L}\right)\right)$ of a rough hypergraph $\mathscr{R}=(\underline{\mathscr{R}}, \overline{\mathscr{R}})$ is the 2 -section of $\mathscr{R}$ with a pair of mappings $L=(\underline{L}, \bar{L})$, where $\underline{L}:\left[\psi[D]_{2} \longrightarrow \underline{\psi} D\right.$ and $\bar{L}:[\bar{\psi} D]_{2} \longrightarrow \bar{\psi} D$ are such that

$$
\begin{aligned}
& \bar{L}(k g)=\{\bar{E} \in \bar{\psi} D \mid k, g \in \bar{E}\}, \\
& \underline{L}(k g)=\{\underline{E} \in \underline{\psi} D \mid k, g \in \underline{E}\} .
\end{aligned}
$$

In other words, the L2-section is a labeled 2-section of a rough hypergraph. As compared to 2-section, L2-section provides additional information to trace back the edges of $[\mathscr{R}]_{2}$ which are associated with the hyperedges of $\mathscr{R}$. Thus, the original rough hypergraph can be easily constructed from the L2-section. The inverse $[\mathscr{R}]_{L 2}^{-1}$ is the rough hypergraph whose L2-section is $[\mathscr{R}]_{L 2}$ with $\psi D=$ $\cup_{k g \in[\psi D]_{2}} \underline{L}(k g)$ and $\bar{\psi} D=\cup_{k g \in[\bar{\psi} D]_{2}} \bar{L}(\mathrm{~kg})$.

Example 2. Consider a rough hypergraph $\mathscr{R}$ shown in Figure 4 . The 2 -section $[\mathscr{R}]_{2}$ of $\mathscr{R}$ is given in Figure 4 with dashed lines and L2-section $[\mathscr{R}]_{L 2}$ is given in Figure 5.

Remark 1 . Let $\mathscr{R}$ be a rough hypergraph; then, Definition 11 directly follows that
(1) $[\mathscr{R}]_{2}^{-1}=\mathscr{R}$
(2) $\left[[\mathscr{R}]_{2}^{-1}\right]_{2}=[\mathscr{R}]_{2}$

Lemma 1. Let $\mathscr{R}_{1}$ and $\mathscr{R}_{2}$ be two isomorphic rough hypergraphs; then, $\left[\mathscr{R}_{1}\right]_{2} \cong\left[\mathscr{R}_{2}\right]_{2}$ and $\left[\mathscr{R}_{1}\right]_{L 2} \cong\left[\mathscr{R}_{2}\right]_{L 2}$.

Proof. Let $\mathscr{R}_{1}=\left(\left(\varphi A_{1}, \psi D_{1}\right),\left(\bar{\varphi} A_{1}, \bar{\psi} D_{1}\right)\right)$ and $\mathscr{R}_{2}=$ $\left(\left(\varphi A_{2}, \psi D_{2}\right),\left(\bar{\varphi} A_{2}, \bar{\psi} \bar{D}_{2}\right)\right)$ be two rough hypergraphs, then $\left.\left.\mathscr{R}_{1}\right]_{2}=T\left(\varphi A_{1},\left[\psi D_{1}\right]_{2}\right),\left(\bar{\varphi} A_{1},\left[\bar{\psi} D_{1}\right]_{2}\right)\right)$ and $\left[\mathscr{R}_{2}\right]_{2}=$ $\left(\left(\varphi A_{2},\left[\psi \bar{D}_{2}\right]_{2}\right),\left(\bar{\varphi} A_{2},\left[\bar{\psi} D_{2}\right]_{2}\right)\right)$. The vertex set of $\mathscr{R}_{j}$ and $\left[\overline{\mathscr{R}}_{j}\right]_{2}$ is the same for $j=1,2$. Let $\mathrm{kg} \in\left[\psi \mathrm{D}_{1}\right]_{2}$; then, there exists $\underline{E} \in \psi D_{1}$ such that $\{k, g\} \subseteq \underline{E}$. Since $\mathscr{R}_{1} \cong \mathscr{R}_{2}$, therefore $f: \mathscr{R}_{1} \longrightarrow \mathscr{R}_{2}$ is an isomorphism and $f(\underline{E})$ is a hyperedge in $\mathscr{R}_{2}$ such that $\{f(k), f(g)\} \in f(\underline{E})$, hence, $f(k) f(g)$ in an edge in $\left[\mathscr{R}_{2}\right]_{2}$ and so $\left[\mathscr{R}_{1}\right]_{2} \cong\left[\mathscr{R}_{2}\right]_{2}$.

Let $\left[\mathscr{R}_{1}\right]_{L 2}=\left(\left(\varphi A_{1},\left[\psi D_{1}\right]_{2}, \underline{L}_{1}\right),\left(\bar{\varphi} A_{1},\left[\bar{\psi} D_{1}\right]_{2}, \bar{L}_{1}\right)\right)$ and $\left[\mathscr{R}_{2}\right]_{L 2}=\left(\left(\varphi A_{2},\left[\bar{\psi} D_{2}\right]_{2}, \bar{L}_{2}\right),\left(\bar{\varphi} A_{2},\left[\bar{\psi} D_{2}\right]_{2}, \bar{L}_{2}\right)\right)$. It remains to show that the labeling functions $L_{1}=\left(\underline{L}_{1}, \bar{L}_{1}\right)$ and $L_{2}=$ $\left(\underline{L}_{2}, \bar{L}_{2}\right)$ are equal. Clearly, for any $k_{1} g_{1} \in\left[\psi D_{1}\right]_{2}$, $\underline{L}_{1}=\left\{\underline{E}_{1} \mid k_{1}, g_{1} \in \underline{E}_{1}\right\}=\{f(E) \mid f(k), f(g) \in f(\underline{E})\}=\underline{L}_{2}$.

Similarly, $\bar{L}_{1}=\bar{L}_{2}$. Hence, the labeling functions $L_{1}$ and $L_{2}$ are equal and $\left[\mathscr{R}_{1}\right]_{L 2} \cong\left[\mathscr{R}_{2}\right]_{L 2}$.

Definition 12 . Let $\mathscr{R}$ be a rough hypergraph on $Q$; then, the distance between any two vertices $k$ and $g$ is defined as

$$
d_{\mathscr{R}}(k, g)=\frac{1}{2}\left[d_{\underline{\mathscr{R}}}(k, g)+d_{\overline{\mathscr{R}}}(k, g)\right]
$$




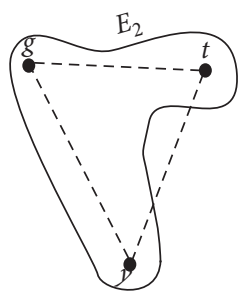

FIGURE 4: 2-section $((\varphi A, \psi D),(\bar{\varphi} A, \bar{\psi} D))$.
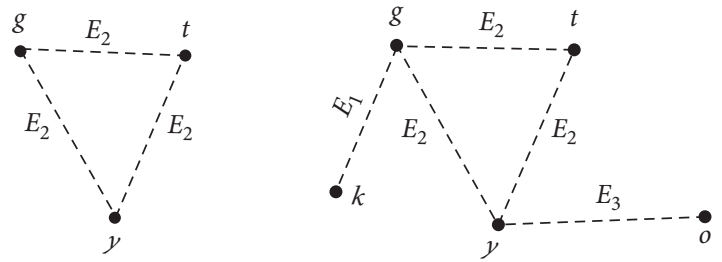

FigURE 5: L2-section of rough hypergraph $((\underline{\varphi} A, \underline{\psi} D),(\bar{\varphi} A, \bar{\psi} D))$.

where $d_{\mathscr{R}}(k, g)$ and $d_{\overline{\mathscr{R}}}(k, g)$ are lengths of shortest hyperpaths between $k$ and $g$ in $\underline{\mathscr{R}}$ and $\mathscr{\mathscr { R }}$, respectively.

Lemma 2. Let $\mathscr{R}$ be a rough hypergraph on $\mathrm{Q}$; then, for any $k, g \in Q d_{\mathscr{R}}(k, g)=d_{[\mathscr{R}]_{2}}(k, g)$.

Proof. If $k$ and $g$ are in different connected components in $\mathscr{R}$ or $\overline{\mathscr{R}}$, or both, then clearly $k$ and $g$ are in different components in $[\underline{\mathscr{R}}]_{2}$ or $[\overline{\mathscr{R}}]_{2}$. In this case, $\mathscr{R}=\overline{\mathscr{R}}=\infty$. Assume that $\mathscr{R}$ is a connected rough hypergraph; then, $\mathscr{R}$ and $\overline{\mathscr{R}}$ are both connected hypergraphs and so is $[\mathscr{R}]_{2}$. Let $k, \underline{E}_{1}, k_{1}, \underline{E}_{2}, \ldots, k_{s-1}, \underline{E}_{s}, g$ be the shortest hyperpath in $\underline{R}$ between vertices $k$ and $g$. Then, by the construction of $\left[\mathscr{R}_{2}\right]_{2}$, there exists a hyperwalk in $k, \underline{e}_{1}, k_{1}, \underline{e}_{2}, \ldots, k_{s-1}, \underline{e}_{s}, k_{s}=g$ in $[\underline{\mathscr{R}}]_{2}$. Let $k, \underline{f}_{1}, k_{1}, \underline{f}_{2}, \ldots, k_{p-1}, \underline{f}_{p}, g$ be the shortest path in $\underline{\mathscr{R}}$. Clearly $s \geq p$. Thus, corresponding to every $\underline{f}_{j} \in[\underline{\psi} D]_{2}$, there exists $\underline{F}_{j} \in \underline{\psi} \underline{\mathrm{D}}$ such that $\underline{f}_{j} \subseteq \underline{F}_{j}$, and so a hyperwalk of length $p$ in $\mathscr{R}$. A contradiction, hence $d_{\mathscr{\mathscr { R }}}(k, g)=d_{[\mathscr{R}]_{2}}(k, g)$. Similarly, $d_{\overline{\mathscr{R}}}(k, g)=d_{[\overline{\mathscr{R}}]_{2}}(k, g)$. It clearly follows that $d_{\mathscr{R}}(k, g)=d_{[\mathscr{R}]_{2}}(k, g)$.

We now study certain products of rough hypergraphs. In each product, the vertex set is the Cartesian product of the sets of vertices of all rough hypergraphs. The adjacency of edges is based on the adjacency properties defined in the product. Let $\mathscr{R}_{1} \circledast \mathscr{R}_{2}$ denote any product of two rough hypergraphs $\mathscr{R}_{1}$ and $\mathscr{R}_{2}$. For any rough hypergraph $\mathscr{R}$, if there exists another rough hypergraph $\mathscr{U}$ such that $\mathscr{R} \circledast \mathscr{U}=\mathscr{R}$, then $\mathscr{U}$ is called the unit element. Note that $\mathcal{U}$ must be a hypergraph with a single vertex and no loops. A rough hypergraph $\mathscr{R}$ is called prime if whenever $\mathscr{R}=\mathscr{R}_{1} \circledast \mathscr{R}_{2}$, then either $\mathscr{R}_{1}=\mathscr{U}$ or $\mathscr{R}_{2}=\mathscr{U}$.

Definition 13. Let $\mathscr{R}$ be a rough hypergraph on $Q$. The rough line graph of $\mathscr{R}$ is a rough graph $L(\mathscr{R})=(L(\underline{\mathscr{R}}), L(\overline{\mathscr{R}}))$ such that
(1) $L(\mathscr{J})=\left(\varphi A_{L}, \psi D_{L}\right)$, where $\varphi A_{L}=\psi D$. That is, the hyperedge set of $\mathscr{R}=(\varphi A, \bar{\psi} D)$ is the vertex set of $L(\underline{R})$. For any $E_{i}, E_{k} \bar{\epsilon} \psi \bar{D}$, if $E_{i} \cap E_{k} \neq \varnothing$, then $E_{i} E_{k} \in \psi D_{L}$.

(2) $L(\overline{\mathcal{J}})=\left(\bar{\varphi} A_{L}, \bar{\psi} D_{L}\right)$, where $\bar{\varphi} A_{L}=\bar{\psi} D$. That is, the hyperedge set of $\mathscr{\mathscr { R }}=(\bar{\varphi} A, \bar{\psi} D)$ is the vertex set of $L(\bar{R})$. For any $E_{i}, E_{k} \in \bar{\psi} D$, if $E_{i} \cap E_{k} \neq \varnothing$, then $E_{i} E_{k} \in \bar{\psi} D_{L}$.

Example 3. The rough line graph $L(\mathscr{R})$ of Figure 3 is shown with dashed lines in Figure 6.

Definition 14. A rough hypergraph $\mathscr{R}$ is called connected if $\underline{\mathscr{R}}$ and $\overline{\mathscr{R}}$ are both connected hypergraphs.

Lemma 3. A rough hypergraph $\mathscr{R}$ is connected if and only if $L(\mathscr{R})$ is a connected rough graph.

Definition 15. A rough hypergraph $\mathscr{R}=((\varphi A$, $\psi D),(\bar{\varphi} A, \bar{\psi} D))$ is called linear if $\underline{\mathscr{R}}=(\varphi A, \psi D)$ and $\overline{\mathscr{R}}=$ $\overline{(\varphi} A, \bar{\psi} D)$ are linear hypergraphs, that is,

(1) For any two hyperedges $\underline{E}_{i}, \underline{E}_{j} \in \psi D$,

(a) $\underline{E}_{i} \subseteq \underline{E}_{j} \Rightarrow i=j$

(b) $\left|\underline{E}_{i} \cap \underline{E}_{j}\right| \leq 1$

(2) For any two hyperedges $\bar{E}_{i}, \bar{E}_{j} \in \bar{\psi} D$,

(a) $\bar{E}_{i} \subseteq \bar{E}_{j} \Rightarrow i=j$

(b) $\left|\bar{E}_{i} \cap \vec{E}_{j}\right| \leq 1$

Theorem 1. Any nontrivial simple rough graph is a rough line graph of a linear rough hypergraph.

Proof. Let $\mathscr{G}=((\varphi C, \psi B),(\bar{\varphi} C, \bar{\psi} B))$ be a rough graph on $Q$. Assume, without loss of generality, that $\mathscr{U}$ is a connected rough graph without multiple edges. A rough hypergraph $\mathscr{R}=((\underline{\alpha}, \beta),(\bar{\alpha}, \bar{\beta}))$ can be constructed from $\mathcal{U}$ as follows:

(1) The vertex set of $\mathscr{R}$ is the edge set of $\mathscr{G}$, that is, $\underline{\alpha}=$ $\psi B$ and $\bar{\alpha}=\psi B$

(2) Let $Q=\left\{k_{1}, \bar{k}_{2}, \ldots, k_{m}\right\}$, then,

(a) If $\underline{E}_{i}$ is the collection of those edges of $(\varphi C, \psi B)$ which has $k_{i}$ as incidence vertex, then $E_{i}^{-} \in \bar{\beta}$ is a hyperedge in $(\underline{\alpha}, \underline{\beta})$; that is, $\underline{E}_{i}=\left\{k_{i} k_{j} \in \underline{\psi}\right.$ $\left.B \mid k_{j} \in Q, j=1,2,3, \ldots\right\}$

(b) If $\vec{E}_{i}$ is the collection of those edges of $(\bar{\varphi} C, \bar{\psi} B)$ which has $k_{i}$ as incidence vertex, then $\bar{E}_{i} \in \bar{\beta}$ is a hyperedge in $(\bar{\alpha}, \bar{\beta})$; that is, $\bar{E}_{i}=\left\{k_{i} k_{j} \in \bar{\psi}\right.$ $\left.B \mid k_{j} \in Q, j=1,2,3, \ldots\right\}$

It remains to show that $\mathscr{R}$ is linear. Let $\underline{E}_{i}$ and $\underline{E}_{j}$ be two hyperedges in $(\underline{\alpha}, \beta)$ such that $\underline{E}_{i} \cap \underline{E}_{j}=\left\{e_{r}, e_{t}\right\}$; that is, both the edges $e_{r}$ and $e_{t}$ have two common vertices. Since $(\varphi C, \psi B)$ has no multiple edges, therefore $e_{r}=e_{t}$. Hence, $(\bar{\alpha}, \beta)$ is a linear hypergraph proving that $\mathscr{R}$ is a linear rough hypergraph. 

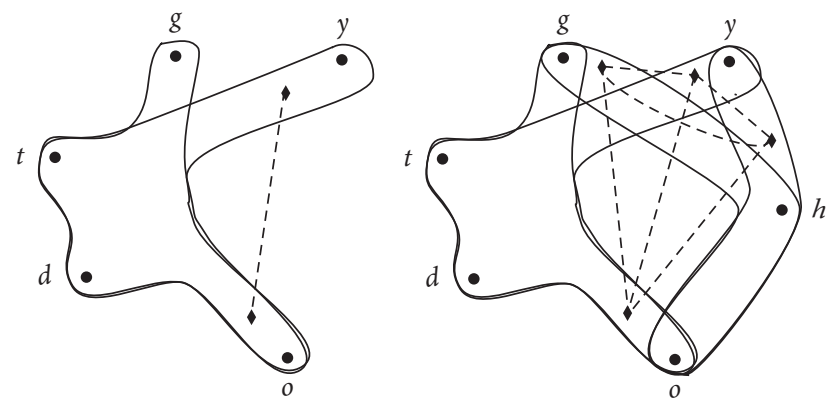

Figure 6: Rough line graph $L(\mathscr{R})$.

Theorem 2. For any rough hypergraph $\mathscr{R}, L(\mathscr{R}) \cong\left[\mathscr{R}^{*}\right]_{2}$.

Proof. Let $\mathscr{R}=((\varphi A, \psi D),(\bar{\varphi} A, \bar{\psi} D))$ be a rough hypergraph on $Q=\left\{k_{1}, \bar{k}_{2}, \ldots, k_{n}\right\}$ and $\psi D=\left\{\underline{E}_{1}, \underline{E}_{2}, \ldots, \underline{E}_{m}\right\}$, $\bar{\psi} D=\left\{\bar{E}_{1}, \bar{E}_{2}, \ldots, \bar{E}_{m}\right\}$. The hyperedge set of $\mathscr{R}$ is the vertex set of $L(\mathscr{R})$ which is also the vertex set of $\left[\mathscr{R}^{*}\right]_{2}$. Let $\left\{\underline{X}_{1}, \underline{X}_{2}, \ldots, \underline{X}_{n}\right\}$ be the hyperedge set of $\underline{\mathscr{R}}^{*}$ such that $\underline{X}_{i}=\left\{\underline{E}_{j} \mid k_{i} \in \underline{E}_{j}\right\}$; then, $\left\{E_{l} E_{j} \mid E_{l}, E_{j} \in X_{i}\right\}$ is the edge set of $L(\underline{\mathscr{R}})$ and $\left[\underline{\mathscr{R}}^{*}\right]_{2}$. Thus, $L(\underline{\mathscr{R}}) \cong\left[\underline{\mathscr{R}}^{*}\right]_{2}$. On the same argument, $L(\overline{\mathscr{R}}) \cong\left[\overline{\mathscr{R}}^{*}\right]_{2}$. Hence, $L(\mathscr{R}) \cong\left[\mathscr{R}^{*}\right]_{2}$.

Lemma 4. For any rough hypergraph $\mathscr{R}$,

(1) $\mathscr{R}^{* *} \cong \mathscr{R}$

(2) If $\mathscr{R}_{1} \cong \mathscr{R}_{2}$, then $\mathscr{R}_{1}^{*} \cong \mathscr{R}_{2}^{*}$

The proof of Lemma 4 is a direct consequence of Definitions 10 and 28.

Theorem 3. For any rough hypergraph $\mathscr{R}, L\left(\mathscr{R}^{*}\right) \cong[\mathscr{R}]_{2}$.

Proof. By Theorem 2 and Lemma 4, $[\mathscr{R}]_{2} \cong$ $\left[\mathscr{R}^{* *}\right]_{2} \cong L\left(\mathscr{R}^{*}\right)$.

Theorem 4. If $\mathscr{R}=((\varphi A, \psi D),(\bar{\varphi} A, \bar{\psi} D))$ is a linear rough hypergraph, then $\mathscr{R}^{*}=\left(\left(\underline{\varphi} A^{*}, \underline{\psi} D^{*}\right),\left(\bar{\varphi} A^{*}, \bar{\psi} D^{*}\right)\right)$ is also linear.

Proof. Since $\mathscr{R}$ is linear, therefore $(\varphi A, \psi D)$ and $(\bar{\varphi} A, \bar{\psi} D)$ are linear hypergraphs. On the contrary, suppose that $\left(\varphi A^{*}, \psi D^{*}\right)$ is not linear. Then, there exist hyperedges $\underline{X}_{i}$ and $\underline{X}_{j}$ in $\left(\underline{\varphi} A^{*}, \underline{\psi} D^{*}\right)$ such that $\left|\underline{X}_{i} \cap \underline{X}_{j}\right|=2$. Let $\underline{X}_{i} \cap \underline{X}_{j}=\left\{E_{t}, E_{s}\right\}$. Definition 28 implies that $E_{t}$ and $E_{s}$ have two common vertices $k_{i}, k_{j}$, that is, $k_{i}, k_{j} \in E_{t}$ and $k_{i}, k_{j} \in E_{s}$. It denies the linearity of $\underline{\mathscr{R}}$. Thus, $\mathscr{J}^{*}$ is linear. Following similar arguments, the linearity of $\overline{\mathscr{F}}^{*}$ can be proved. Hence, $\mathscr{J}^{*}$ is a linear rough hypergraph.

3.1. Cartesian Product. In this subsection, we introduce the concept of Cartesian product in rough hypergraphs and study its 2-section, L2-section, distance, covering, and coloring of the Cartesian product of rough hypergraphs.
Definition 16. Let $\mathscr{R}_{1}=\left(\mathscr{R}_{1}, \overline{\mathscr{R}}_{1}\right)$ and $\mathscr{R}_{2}=\left(\mathscr{R}_{2}, \overline{\mathscr{R}}_{2}\right)$ be two rough hypergraphs. The Cartesian product of $\mathscr{R}_{1}$ and $\mathscr{R}_{2}$ is a rough hypergraph $\mathscr{R}_{1} \square \mathscr{R}_{2}=\left(\underline{\mathscr{R}}_{1} \square \mathscr{R}_{2}, \overline{\mathscr{R}}_{1} \square \overline{\mathscr{R}}_{2}\right)$ which is defined as

(1) $\underline{\mathscr{R}}_{1} \square \underline{\mathscr{R}}_{2}=\left(\underline{\varphi} A_{1} \times \underline{\varphi} A_{2}, \underline{\psi} D_{1} \square \underline{\psi} D_{2}\right)$

(a) $\underline{\varphi} A_{1} \times \underline{\varphi} A_{2}=\left\{\left(k_{1}, k_{2}\right) \mid k_{1} \in \underline{\varphi} A_{1}, k_{2} \in \underline{\varphi} A_{2}\right\}$

(b) $\psi D_{1} \square \psi D_{2}=\left\{\left\{k_{1}\right\} \times \underline{E}_{2} \mid k_{1} \in \varphi \quad A_{1}, \underline{E}_{2} \in \psi D_{2}\right\}$ $\bar{\cup}\left\{\underline{E}_{1} \times\left\{k_{2}\right\} \mid \underline{E}_{1} \in \psi D_{1}, k_{2} \in \bar{\varphi} A_{2}\right\}$

(2) $\overline{\mathscr{R}}_{1} \square \overline{\mathscr{R}}_{2}=\left(\bar{\varphi} A_{1} \times \bar{\varphi} A_{2}, \bar{\psi} D_{1} \square \bar{\psi} D_{2}\right)$

(a) $\bar{\varphi} A_{1} \times \bar{\varphi} A_{2}=\left\{\left(k_{1}, k_{2}\right) \mid k_{1} \in \bar{\varphi} A_{1}, k_{2} \in \bar{\varphi} A_{2}\right\}$

(b) $\bar{\psi} D_{1} \square \bar{\psi} D_{2}=\left\{\left\{k_{1}\right\} \times \bar{E}_{2} \mid k_{1} \in \bar{\varphi} \quad A_{1}, \bar{E}_{2} \in \bar{\psi} D_{2}\right\}$ $\cup\left\{\bar{E}_{1} \times\left\{k_{2}\right\} \mid \bar{E}_{1} \in \bar{\psi} D_{1}, k_{2} \in \bar{\varphi} A_{2}\right\}$

In short, $\underline{\mathscr{R}}_{1} \square \underline{\mathscr{R}}_{2}$ and $\overline{\mathscr{R}}_{1} \square \overline{\mathscr{R}}_{2}$ are the Cartesian products of lower approximate hypergraphs $\mathscr{R}_{1}, \mathscr{R}_{2}$ and upper approximate hypergraphs $\overline{\mathscr{R}}_{1}, \overline{\mathscr{R}}_{2}$, respectively. Just like the Cartesian product of hypergraphs, the Cartesian product of rough hypergraphs is associative, distributive with respect to the disjoint union, commutative, and a unit as a trivial rough hypergraph with a single vertex. That is, for any rough hypergraphs $\mathscr{R}, \mathscr{R}_{1}, \mathscr{R}_{2}$, and $\mathscr{R}_{3}$, the following properties hold:

(1) $\mathscr{R}_{1} \square\left(\mathscr{R}_{2} \square \mathscr{R}_{3}\right) \cong\left(\mathscr{R}_{1} \square \mathscr{R}_{2}\right) \square \mathscr{R}_{3}$

(2) $\mathscr{R}_{1} \square \mathscr{R}_{2} \cong \mathscr{R}_{2} \square \mathscr{R}_{1}$

(3) $\mathscr{R}_{1} \square\left(\mathscr{R}_{2} \cup \mathscr{R}_{3}\right) \cong\left(\mathscr{R}_{1} \square \mathscr{R}_{2}\right) \cup\left(\mathscr{R}_{1} \square \mathscr{R}_{2}\right)$

(4) $\mathscr{R} \square \mathscr{U} \cong \mathscr{R}$, where $\mathscr{U}$ is a single vertex rough hypergraph without loops

Theorem 5. Let $\mathscr{R}_{1}$ and $\mathscr{R}_{2}$ be two rough hypergraphs; then,

(1) $r\left(\mathscr{R}_{1} \square \mathscr{R}_{2}\right)=\max \left\{r\left(\mathscr{R}_{1}\right), r\left(\mathscr{R}_{2}\right),\left(r\left(\mathscr{R}_{1}\right)+\right.\right.$ $\left.\left.r\left(\overline{\mathscr{R}}_{2}\right) / 2\right),\left(r\left(\underline{\mathscr{R}}_{2}\right)+r\left(\overline{\mathscr{R}}_{1}\right) / 2\right)\right\}$

(2) $s\left(\mathscr{R}_{1} \square \mathscr{R}_{2}\right)=\min \left\{s\left(\mathscr{R}_{1}\right), s\left(\mathscr{R}_{2}\right)\right.$, $\left.\left(s\left(\underline{\mathscr{R}}_{1}\right)+s\left(\overline{\mathscr{R}}_{2}\right) / 2\right),\left(s\left(\underline{\mathscr{R}}_{2}\right)+s\left(\overline{\mathscr{R}}_{1}\right) / 2\right)\right\}$

Proof. Since, by Definition 16, $\mathscr{R}_{1} \square \mathscr{R}_{2}=\left(\mathscr{R}_{1} \square \mathscr{R}_{2}\right.$, $\left.\overline{\mathscr{R}}_{1} \square \overline{\mathscr{R}}_{2}\right)$, we first need to compute $r\left(\underline{\mathscr{R}}_{1} \square \mathscr{\mathscr { R }}_{2}\right)$ and $r\left(\mathscr{\mathscr { R }}_{1} \square \overline{\mathscr{R}}_{2}\right)$. By Definition 6,

$$
\begin{aligned}
r\left(\underline{\mathscr{R}}_{1} \square \underline{\mathscr{R}}_{2}\right)= & \max _{E \in \underline{\psi} D_{1} \square \underline{D_{2}}}|E| \\
= & \max \left\{\left|\left\{k_{1}\right\} \times \underline{E}_{2}\right| \mid k_{1} \in \underline{\varphi} A_{1}, \underline{E}_{2} \in \underline{\psi} D_{2}\right\} \\
& \cup\left\{\left|\underline{E}_{1} \times\left\{k_{2}\right\}\right| \mid \underline{E}_{1} \in \underline{\psi} D_{1}, k_{2} \in \underline{\varphi} A_{2}\right\} \\
= & \max \left\{\left|\underline{E}_{2}\right| \mid \underline{E}_{2} \in \underline{\psi} D_{2}\right\} \cup\left\{\left|\underline{E}_{1}\right| \mid \underline{E}_{1} \in \underline{\psi} D_{1}\right\} \\
= & \max \left\{\max _{\underline{E}_{1} \in \underline{\psi} D_{1}}\left|\underline{E}_{1}\right|, \max _{\underline{E}_{2} \in \underline{\underline{D}} D_{2}}\left|\underline{E}_{2}\right|\right\} \\
& \Rightarrow r\left(\underline{\mathscr{R}}_{1} \square \underline{\mathscr{R}}_{2}\right)=\max \left\{r\left(\underline{\mathscr{R}}_{1}\right), r\left(\underline{\mathscr{R}}_{2}\right)\right\} .
\end{aligned}
$$

Similarly, $r\left(\overline{\mathscr{R}}_{1} \square \overline{\mathscr{R}}_{2}\right)=\max \left\{r\left(\overline{\mathscr{R}}_{1}\right), r\left(\overline{\mathscr{R}}_{2}\right)\right\}$. Hence, 


$$
\begin{aligned}
r\left(\mathscr{R}_{1} \square \mathscr{R}_{2}\right) & =\frac{1}{2}\left\{\max \left\{r\left(\underline{\mathscr{R}}_{1}\right), r\left(\underline{\mathscr{R}}_{2}\right)\right\}+\max \left(\overline{\mathscr{R}}_{1}\right), r\left(\overline{\mathscr{R}}_{2}\right)\right\} \\
& =\max \left\{\frac{r\left(\underline{\mathscr{R}}_{1}\right)+r\left(\overline{\mathscr{R}}_{1}\right)}{2}, \frac{r\left(\underline{\mathscr{R}}_{2}\right)+r\left(\overline{\mathscr{R}}_{2}\right)}{2}, \frac{r\left(\underline{\mathscr{R}}_{1}\right)+r\left(\overline{\mathscr{R}}_{2}\right)}{2}, \frac{r\left(\mathscr{\mathscr { R }}_{2}\right)+r\left(\overline{\mathscr{R}}_{1}\right)}{2}\right\} \\
& =\max \left\{r\left(\mathscr{R}_{1}\right), r\left(\mathscr{R}_{2}\right), \frac{r\left(\mathscr{R}_{1}\right)+r\left(\overline{\mathscr{R}}_{2}\right)}{2}, \frac{r\left(\mathscr{R}_{2}\right)+r\left(\overline{\mathscr{R}}_{1}\right)}{2}\right\} .
\end{aligned}
$$

By Definition 6, the antirank of $\mathscr{R}_{1} \square \mathscr{R}_{2}$ is given as Similarly, $s\left(\overline{\mathscr{R}}_{1} \square \overline{\mathscr{R}}_{2}\right)=\min \left\{s\left(\overline{\mathscr{R}}_{1}\right), s\left(\overline{\mathscr{R}}_{2}\right)\right\}$. Hence,

$$
\begin{aligned}
& s\left(\underline{\mathscr{R}}_{1} \square \underline{\mathscr{R}}_{2}\right)=\min _{E \in \underline{\psi} D_{1} \square \underline{\psi} D_{2}}|E| \\
& =\min \left\{\left|\left\{k_{1}\right\} \times \underline{E}_{2}\right| \mid k_{1} \in \underline{\varphi} A_{1}, \underline{E}_{2} \in \underline{\psi} D_{2}\right\} \\
& \cup\left\{\left|\underline{E}_{1} \times\left\{k_{2}\right\}\right| \mid \underline{E}_{1} \in \underline{\psi} D_{1}, k_{2} \in \underline{\varphi} A_{2}\right\} \\
& =\min \left\{\left|\underline{E}_{2}\right| \mid \underline{E}_{2} \in \underline{\psi} D_{2}\right\} \cup\left\{\left|\underline{E}_{1}\right| \mid \underline{E}_{1} \in \underline{\psi} D_{1}\right\} \\
& =\min \left\{\min _{\underline{E}_{1} \in \underline{\psi} D_{1}}\left|\underline{E}_{1}\right|, \min _{\underline{E}_{2} \in \underline{\psi} \underline{D}_{2}}\left|\underline{E}_{2}\right|\right\} \\
& \Rightarrow s\left(\underline{\mathscr{R}}_{1} \square \underline{\mathscr{R}}_{2}\right)=\min \left\{s\left(\underline{\mathscr{R}}_{1}\right), s\left(\underline{\mathscr{R}}_{2}\right)\right\} \text {. }
\end{aligned}
$$

$$
\begin{aligned}
s\left(\mathscr{R}_{1} \square \mathscr{R}_{2}\right) & =\frac{1}{2}\left\{\min \left\{s\left(\underline{\mathscr{R}}_{1}\right), s\left(\underline{\mathscr{R}}_{2}\right)\right\}+\min s\left(\overline{\mathscr{R}}_{1}\right), s\left(\overline{\mathscr{R}}_{2}\right)\right\} \\
& =\min \left\{\frac{s\left(\underline{\mathscr{R}}_{1}\right)+s\left(\overline{\mathscr{R}}_{1}\right)}{2}, \frac{s\left(\underline{\mathscr{R}}_{2}\right)+s\left(\overline{\mathscr{R}}_{2}\right)}{2}, \frac{s\left(\underline{\mathscr{R}}_{1}\right)+s\left(\overline{\mathscr{R}}_{2}\right)}{2}, \frac{s\left(\mathscr{R}_{2}\right)+s\left(\overline{\mathscr{R}}_{1}\right)}{2}\right\} \\
& =\min \left\{s\left(\mathscr{R}_{1}\right), s\left(\mathscr{R}_{2}\right), \frac{s\left(\mathscr{R}_{1}\right)+s\left(\overline{\mathscr{R}}_{2}\right)}{2}, \frac{s\left(\mathscr{\mathscr { R }}_{2}\right)+s\left(\overline{\mathscr{R}}_{1}\right)}{2}\right\} .
\end{aligned}
$$

Lemma 5. Let $\mathscr{R}_{1}$ and $\mathscr{R}_{2}$ be two rough hypergraphs, then $\left[\mathscr{R}_{1} \square \mathscr{R}_{2}\right]_{2}=\left[\mathscr{R}_{1}\right]_{2} \square\left[\mathscr{R}_{2}\right]_{2}$.

Proof. Since the vertex set of $\mathscr{R}_{i}$ and $\left[\mathscr{R}_{i}\right]_{2}$ is the same for $i=1,2$, therefore the vertex set of $\left[\mathscr{R}_{1} \square \mathscr{R}_{2}\right]_{2}$ and $\left[\mathscr{R}_{1}\right]_{2} \square\left[\mathscr{R}_{2}\right]_{2}$ is the same. It only needs to show that the set of hyperedges of $\left[\mathscr{R}_{1} \square \mathscr{R}_{2}\right]_{2}$ and $\left[\mathscr{R}_{1}\right]_{2} \square\left[\mathscr{R}_{2}\right]_{2}$ is the same. By Definition 16, $\left[\mathscr{R}_{1} \square \mathscr{R}_{2}\right]_{2}=\left(\left[\underline{\mathscr{R}}_{1} \square \mathscr{R}_{2}\right]_{2},\left[\overline{\mathscr{R}}_{1} \square \overline{\mathscr{R}}_{2}\right]_{2}\right)$, where $\quad\left[\mathscr{R}_{1} \square \mathscr{R}_{2}\right]_{2}=\left(\varphi A_{1} \times \varphi A_{2},\left[\psi D_{1} \square \psi D_{2}\right]_{2}\right) \quad$ and $\left[\overline{\mathscr{R}}_{1} \square \overline{\mathscr{R}}_{2}\right]_{2}=\left(\bar{\varphi} A_{1} \times \bar{\varphi} A_{2},\left[\bar{\psi} D_{1} \square \bar{\psi} D_{2}\right]_{2}\right)$. So, we have

$$
\begin{aligned}
{\left[\underline{\psi} D_{1} \square \underline{\psi} D_{2}\right]_{2}=} & \left\{\left(x_{1}, x_{2}\right)\left(y_{1}, y_{2}\right) \mid\left\{\left(x_{1}, x_{2}\right),\left(y_{1}, y_{2}\right)\right\} \subseteq \underline{E} \in \underline{\psi} D_{1} \square \underline{\psi} D_{2}\right\} \\
= & \left\{\left(x_{1}, x_{2}\right)\left(x_{1}, y_{2}\right) \mid y_{1}=x_{1},\left\{x_{2}, y_{2}\right\} \subseteq \underline{E}_{2} \in \underline{\psi} D_{2}\right\} \\
& \cup\left\{\left(x_{1}, x_{2}\right)\left(y_{1}, x_{2}\right) \mid y_{2}=x_{2},\left\{x_{1}, y_{1}\right\} \subseteq \underline{E}_{1} \in \underline{\psi} D_{1}\right\} \\
= & \left\{\left(x_{1}, x_{2}\right)\left(x_{1}, y_{2}\right) \mid x_{1} \in \underline{\varphi} A_{1}, x_{2} y_{2} \in\left[\underline{\psi} D_{2}\right]_{2}\right\} \cup\left\{\left(x_{1}, x_{2}\right)\left(y_{1}, x_{2}\right) \mid x_{1} y_{1} \in\left[\underline{\psi} D_{1}\right]_{2}, x_{2} \in \underline{\varphi} A_{2}\right\} \\
& \Rightarrow\left[\underline{\psi} D_{1} \square \underline{\psi} D_{2}\right]_{2}=\left[\underline{\psi} D_{1}\right]_{2} \square\left[\underline{\psi} D_{2}\right]_{2} .
\end{aligned}
$$


As the vertex set $\left[\underline{\mathscr{R}}_{1} \square \underline{\mathscr{R}}_{2}\right]_{2}$ and $\left[\underline{\mathscr{R}}_{1}\right]_{2} \square\left[\underline{\mathscr{R}}_{2}\right]_{2}$ is also same, so $\left[\underline{\mathscr{R}}_{1} \square \underline{\mathscr{R}}_{2}\right]_{2}=\left[\underline{\mathscr{R}}_{1}\right]_{2} \square\left[\underline{\mathscr{R}}_{2}\right]_{2}$. Similarly, $\quad\left[\overline{\mathscr{R}}_{1}\right.$ $\left.\square \overline{\mathscr{R}}_{2}\right]_{2}=\left[\overline{\mathscr{R}}_{1}\right]_{2} \square\left[\overline{\mathscr{R}}_{2}\right]_{2}$. Hence, $\left[\mathscr{R}_{1} \square \mathscr{R}_{2}\right]_{2}=\left[\mathscr{R}_{1}\right]_{2} \square$ $\left[\mathscr{R}_{2}\right]_{2}$.

Definition 17. Let $\left[\mathscr{R}_{1}\right]_{L 2}=\left(\left(\varphi A_{1},\left[\psi D_{1}\right]_{2}, \underline{L}_{1}\right),\left(\bar{\varphi} A_{1}\right.\right.$, $\left.\left.\left[\bar{\psi} D_{1}\right]_{2}, \bar{L}_{1}\right)\right) \quad$ and $\quad\left[\mathscr{R}_{2}\right]_{L 2}=\left(\left(\bar{\varphi} A_{2},\left[\psi D_{2}\right]_{2}, \underline{L}_{2}\right)\right.$, $\left.\left(\bar{\varphi} A_{2},\left[\bar{\psi} D_{2}\right]_{2}, \bar{L}_{2}\right)\right)$ be L2-sections of two hypergraphs $\mathscr{R}_{1}$ and $\mathscr{R}_{2}$. The Cartesian product of L2-sections is a rough hypergraph with a labeling function $L_{1} \square L_{2}=\left(\underline{L}_{1} \square\right.$ $\left.\underline{L}_{2}, \bar{L}_{1} \square \bar{L}_{2}\right)$, where $\underline{L}_{1} \square \underline{L}_{2}:\left[\psi D_{1}\right]_{2} \times\left[\psi D_{2}\right]_{2} \longrightarrow \psi D_{1} \square \psi \underline{\psi} D_{2}$ and $\bar{L}_{1} \square \bar{L}_{2}:\left[\bar{\psi} D_{1}\right]_{2} \times\left[\bar{\psi} D_{2}\right]_{2} \longrightarrow \bar{\psi} \bar{D}_{1} \square \bar{\psi} D_{2}$ are defined as

$$
\begin{aligned}
& \underline{L}_{1} \square \underline{L}_{2}\left(\left\{k_{1}\right\} \times\left\{l_{2}, g_{2}\right\}\right)=\left\{\left\{k_{1}\right\} \times \underline{E}_{2} \mid \underline{E}_{2} \in \underline{L}_{2}\left(l_{2} g_{2}\right)\right\} \\
& \underline{L}_{1} \square \underline{L}_{2}\left(\left\{l_{1}, g_{1}\right\} \times\left\{k_{2}\right\}\right)=\left\{\underline{E}_{1} \times\left\{k_{2}\right\} \mid \underline{E}_{1} \in \underline{L}_{1}\left(l_{1} g_{1}\right)\right\} \\
& \bar{L}_{1} \square \bar{L}_{2}\left(\left\{k_{1}\right\} \times\left\{l_{2}, g_{2}\right\}\right)=\left\{\left\{k_{1}\right\} \times \bar{E}_{2} \mid \bar{E}_{2} \in \bar{L}_{2}\left(l_{2} g_{2}\right)\right\} \\
& \bar{L}_{1} \square \bar{L}_{2}\left(\left\{l_{1}, g_{1}\right\} \times\left\{k_{2}\right\}\right)=\left\{\bar{E}_{1} \times\left\{k_{2}\right\} \mid \bar{E}_{1} \in \bar{L}_{1}\left(l_{1} g_{1}\right)\right\} .
\end{aligned}
$$

(1) $\left[\mathscr{R}_{1} \square \mathscr{R}_{2}\right]_{L 2}=\left[\mathscr{R}_{1}\right]_{L 2} \square\left[\mathscr{R}_{2}\right]_{L 2}$

(2) $\left[\mathscr{R}_{1} \square \mathscr{R}_{2}\right]_{L 2}^{-1}=\left[\mathscr{R}_{1}\right]_{L 2}^{-1} \square\left[\mathscr{R}_{2}\right]_{L 2}^{-1}$

Proof

(1) It is clear from Lemma 7 that $\left[\mathscr{R}_{1} \square \mathscr{R}_{2}\right]_{2}=\left[\mathscr{R}_{1}\right]_{2} \square\left[\mathscr{R}_{2}\right]_{2}$. It only needs to prove that the labeling function of $\left[\mathscr{R}_{1} \square \mathscr{R}_{2}\right]_{L 2}$ and the function $L_{1} \square L_{2}$ of Definition 17 are the same. Let $\left[\mathscr{R}_{1} \square \mathscr{R}_{2}\right]_{L 2}=\left(\left(\varphi A_{1} \times \underline{\varphi} A_{2},\left[\underline{\psi} D_{1} \square \psi D_{2}\right]_{2}, \underline{L}\right)\right.$, $\left.\left(\bar{\varphi} A_{1} \times \bar{\varphi} A_{2},\left[\bar{\psi} D_{1} \square \overline{\bar{\psi}} D_{2}\right]_{2}, \overline{\bar{L}}\right)\right)$ be the L2-section of $\mathscr{R}_{1} \square \mathscr{R}_{2}$ with a labeling function $L=(\underline{L}, \bar{L})$, where $\underline{L}:\left[\psi D_{1} \square \psi D_{2}\right]_{2} \longrightarrow \psi D_{1} \square \psi D_{2}$ and $\bar{L}:\left[\bar{\psi} D_{1} \square\right.$ $\left.\bar{\psi} D_{2}\right]_{2} \longrightarrow \bar{\psi} D_{1} \square \bar{\psi} D_{2}$ are mappings. It is to be shown that $\underline{L}\left(\left(k_{1}, k_{2}\right)\left(g_{1}, g_{2}\right)\right)=\underline{L}_{1} \square \underline{L}_{2} \quad\left(\left(k_{1}, k_{2}\right)\right.$ $\left.\left(g_{1}, g_{2}\right)\right)$ and $\bar{L}\left(\left(k_{1}, k_{2}\right)\left(g_{1}, g_{2}\right)\right)=\bar{L}_{1} \square \bar{L}_{2}\left(\left(k_{1}, k_{2}\right)\right.$ $\left.\left(g_{1}, g_{2}\right)\right)$. By Definitions 11 and 17 ,

Lemma 6. Let $\mathscr{R}_{1}$ and $\mathscr{R}_{2}$ be two rough hypergraphs, then

$$
\begin{aligned}
\underline{L}\left(\left(k_{1}, k_{2}\right)\left(k_{1}, g_{2}\right)\right)= & \left\{\underline{E} \mid\left\{\left(k_{1}, k_{2}\right),\left(k_{1}, g_{2}\right)\right\} \subseteq \underline{E} \in \underline{\psi} D_{1} \square \underline{\psi} D_{2}\right\} \\
= & \left\{\left\{k_{1}\right\} \times \underline{E}_{2} \mid\left\{\left(k_{1}, k_{2}\right),\left(k_{1}, g_{2}\right)\right\} \subseteq\left\{k_{1}\right\} \times \underline{E}_{2} \in \underline{\psi} D_{1} \square \underline{\psi} D_{2}\right\} \\
= & \left\{\left\{k_{1}\right\} \times \underline{E}_{2} \mid\left\{k_{2}, g_{2}\right\} \subseteq \underline{E}_{2} \in \underline{\psi} D_{2}\right\} \\
= & \left\{\left\{k_{1}\right\} \times \underline{E}_{2} \mid \underline{E}_{2} \in \underline{L}_{2}\left(k_{2} g_{2}\right)\right\} \\
& \Rightarrow \underline{L}\left(\left(k_{1}, k_{2}\right)\left(k_{1}, g_{2}\right)\right)=\underline{L}_{1} \square \underline{L}_{2}\left(\left(k_{1}, k_{2}\right)\left(k_{1}, g_{2}\right)\right) . \\
& \Rightarrow \underline{L}\left(\left(k_{1}, k_{2}\right)\left(g_{1}, k_{2}\right)\right)=\left\{\underline{E} \mid\left\{\left(k_{1}, k_{2}\right),\left(g_{1}, k_{2}\right)\right\} \subseteq \underline{E} \in \underline{\psi} D_{1} \square \underline{\psi} D_{2}\right\} \\
= & \left\{\underline{E}_{1} \times\left\{k_{2}\right\} \mid\left\{\left(k_{1}, k_{2}\right),\left(g_{1}, k_{2}\right)\right\} \subseteq \underline{E}_{1} \times\left\{k_{2}\right\} \in \underline{\psi} D_{1} \square \underline{\psi} D_{2}\right\} \\
= & \left\{\underline{E}_{1} \times\left\{k_{2}\right\} \mid\left\{k_{1}, g_{1}\right\} \subseteq \underline{E}_{1} \in \underline{\psi} D_{1}\right\} \\
= & \left\{\underline{E}_{1} \times\left\{k_{2}\right\} \mid \underline{E}_{1} \in \underline{L}_{1}\left(k_{1} g_{1}\right)\right\} \\
& \Rightarrow \underline{L}\left(\left(k_{1}, k_{2}\right)\left(g_{1}, k_{2}\right)\right)=\underline{L}_{1} \square \underline{L}_{2}\left(\left(k_{1}, k_{2}\right)\left(g_{1}, k_{2}\right)\right) .
\end{aligned}
$$

Thus, $\underline{L}\left(\left(k_{1}, k_{2}\right)\left(g_{1}, g_{2}\right)\right)=\underline{L}_{1} \square \underline{L}_{2}\left(\left(k_{1}, k_{2}\right)\left(g_{1}, g_{2}\right)\right)$, for each $\left(k_{1}, k_{2}\right)\left(g_{1}, g_{2}\right) \in\left[\psi D_{1} \square \psi D_{2}\right]_{2}$. Similarly, $\bar{L}=\bar{L}_{1} \square \bar{L}_{2}$. Hence, $L=L_{1} \square \bar{L}_{2}$ which clearly proves $\left[\mathscr{R}_{1} \square \mathscr{R}_{2}\right]_{L 2}=\left[\mathscr{R}_{1}\right]_{L 2} \square\left[\mathscr{R}_{2}\right]_{L 2}$.

(2) For any rough hypergraph $\mathscr{R},[\mathscr{R}]_{L 2}^{-1}=(\mathscr{R}, L)$ is the rough hypergraph with a labeling function. The proof of part 2 is clear from part 1 and Remark 1. Using Remark 1, $\left[\mathscr{R}_{1} \square \mathscr{R}_{2}\right]_{L 2}^{-1}=\left(\mathscr{R}_{1} \square \mathscr{R}_{2}, L\right)$. By proof of part 1, $\left(\mathscr{R}_{1} \square \mathscr{R}_{2}, L\right)=\left(\mathscr{R}_{1} \square \mathscr{R}_{2}, L_{1} \square L_{2}\right)=$ $\left(\mathscr{R}_{1}, L_{1}\right) \square\left(\mathscr{R}_{2}, L_{2}\right)=\left[\mathscr{R}_{1}\right]_{L 2}^{-1} \square\left[\mathscr{R}_{2}\right]_{L 2}^{-1}$.

Theorem 6. Let $\mathscr{R}_{1}$ and $\mathscr{R}_{2}$ be two rough hypergraphs on $Q_{1}$ and $Q_{2}$; then, for any $k_{1}, g_{1} \in Q_{1}$ and $k_{2}, g_{2} \in Q_{2}$,

$$
d_{\mathscr{R}_{1} \square \mathscr{R}_{2}}\left(\left(k_{1}, k_{2}\right),\left(g_{1}, g_{2}\right)\right)=d_{\mathscr{R}_{1}}\left(k_{1}, g_{1}\right)+d_{\mathscr{R}_{2}}\left(k_{2}, g_{2}\right) \text {. }
$$

Proof. The proof of this theorem is a direct consequence of Proposition 5.1 of [5], Lemmas 2 and 6. Thus, for any two rough hypergraphs $\mathscr{R}_{1}$ and $\mathscr{R}_{2}$,

$$
\begin{aligned}
d_{\mathscr{R}_{1} \square \mathscr{R}_{2}}\left(\left(k_{1}, k_{2}\right),\left(g_{1}, g_{2}\right)\right) & =d_{\left[\mathscr{R}_{1} \square \mathscr{R}_{2}\right]_{2}}\left(\left(k_{1}, k_{2}\right),\left(g_{1}, g_{2}\right)\right) \\
& =d_{\left[\mathscr{R}_{1}\right]_{2} \square\left[\mathscr{R}_{2}\right]_{2}}\left(\left(k_{1}, k_{2}\right),\left(g_{1}, g_{2}\right)\right) \\
& =d_{\left[\mathscr{R}_{1}\right]_{2}}\left(k_{1}, g_{1}\right)+d_{\left[\mathscr{R}_{2}\right]_{2}}\left(k_{2}, g_{2}\right) \\
& \Rightarrow d_{\mathscr{R}_{1} \square \mathscr{R}_{2}}\left(\left(k_{1}, k_{2}\right),\left(g_{1}, g_{2}\right)\right) \\
& =d_{\mathscr{R}_{1}}\left(k_{1}, g_{1}\right)+d_{\mathscr{R}_{2}}\left(k_{2}, g_{2}\right) .
\end{aligned}
$$

Let $\mathscr{R}$ be a rough hypergraph. If there exist prime rough hypergraphs $\mathscr{R}_{1}, \mathscr{R}_{2}, \ldots, \mathscr{R}_{q}$ such that $\mathscr{R} \cong \mathscr{R}_{1} \square \mathscr{R}_{2} \square \ldots \square \mathscr{R}_{q}$, then it is called PFD (prime factor 
decomposition) of $\mathscr{R}$ into $q$ factors w.r.t the Cartesian product.

Remark 2. Every connected rough hypergraph has a unique PFD with respect to the Cartesian product.

The method to obtain a PFD of a rough hypergraph using its L2-section is illustrated in Algorithm 1.

Definition 18. Let $\mathscr{R}_{1}$ and $\mathscr{R}_{2}$ be two rough hypergraphs on $Q_{1}$ and $Q_{2}$, respectively. A homomorphism from $\mathscr{R}_{1}$ into $\mathscr{R}_{2}$ is a mapping $f: Q_{1} \longrightarrow Q_{2}$ if there exist homomorphisms $f: Q_{1} \longrightarrow Q_{2}$ and $\bar{f}: Q_{1} \longrightarrow Q_{2}$; that is,

(1) If $\bar{f}(D)$ is a hyperedge in $\overline{\mathscr{R}_{2}}$, then $D$ is a hyperedge in $\overline{\mathscr{R}_{1}}$

(2) If $f(D)$ is a hyperedge in $\mathscr{R}_{2}$, then $D$ is a hyperedge in $\mathscr{R}_{1}$

A homomorphism which is a one-to-one correspondence between $Q_{1}$ and $Q_{2}$ is called an isomorphism. In this case, we say that the rough hypergraphs $\mathscr{R}_{1}$ and $\mathscr{R}_{2}$ are isomorphic to each other and write as $\mathscr{R}_{1} \cong \mathscr{R}_{2}$.

Definition 19. Let $\mathscr{R}=((\varphi A, \psi D),(\bar{\varphi} A, \bar{\psi} D)) \quad$ and $\mathscr{R}^{\prime}=\left(\left(\varphi A^{\prime}, \psi D^{\prime}\right),\left(\bar{\varphi} A^{\prime}, \bar{\psi} D^{\prime}\right)\right)$ be two rough hypergraphs on $Q$ and $Q^{+}$, respectively. A surjective homomorphism $f: Q^{\prime} \longrightarrow Q$ is called a covering projection if

(1) $\left|\underline{f}^{-1}(k)\right|=\left|\underline{f}^{-1}(\underline{E})\right|=p$, for all $k \in \underline{\varphi} A, E \in \underline{\psi} D$

(2) $\left|\bar{f}^{-1}(k)\right|=\left|\bar{f}^{-1}(\bar{E})\right|=p$, for all $k \in \bar{\varphi} A, E \in \bar{\psi} D$

(3) $\underline{E}^{\prime} \cap \underline{F}^{\prime}=\varnothing$, for all distinct $\underline{E}^{\prime}, \underline{F}^{\prime} \in f^{-1}(\underline{E}), \underline{E} \in \psi D$

(4) $\bar{E}^{\prime} \cap \bar{F}^{\prime}=\varnothing$, for all distinct $\bar{E}^{\prime}, \bar{F}^{\prime} \in f^{-1}(\bar{E}), \bar{E} \in \overline{\bar{\psi}} D$

The rough hypergraph $\mathscr{R}^{\prime}$ is called a $p$-fold covering of $\mathscr{R}$, and $\mathscr{R}$ is called quotient rough hypergraph of $\mathscr{R}^{\prime}$. If $p=2, \mathscr{R}^{\prime}$ is called a double cover of $\mathscr{R}$.

Definition 20. Let $\mathscr{R}=((\varphi A, \psi D),(\bar{\varphi} A, \bar{\psi} D))$ be a rough hypergraph on $Q$. The sets $\underline{S} \subseteq \varphi A$ and $\bar{S} \subseteq \bar{\varphi} A$ are called independent if they contain no hyperedge of $\mathscr{R}$ and $\overline{\mathscr{R}}$, respectively. The cardinalities of the largest independent sets are denoted by $\beta(\underline{R})$ and $\beta(\overline{\mathscr{R}})$ and are called the independence number of $\mathscr{R}$ and $\overline{\mathscr{R}}$, respectively. The value $\beta(\mathscr{R})=(1 / 2)(\beta(\underline{\mathscr{R}})+\beta(\overline{\mathscr{R}})) \quad$ is called independence number of $\mathscr{R}$.

Definition 21. Let $\mathscr{R}=((\varphi A, \psi D),(\bar{\varphi} A, \bar{\psi} D))$ be a rough hypergraph on $Q$. The subsets $\underline{T} \subseteq \varphi A$ and $\bar{T} \subseteq \bar{\varphi} A$ are called covers of $\mathscr{R}$ and $\bar{R}$, respectively, if $\underline{T} \cap \underline{E} \neq \varnothing$ and $\bar{T} \cap \bar{E} \neq \varnothing$, for each $\underline{E} \in \psi D$ and $\bar{E} \in \bar{\psi} D$. The cardinalities of minimal covers are denoted by $\tau(\underline{\mathscr{R}})$ and $\tau(\overline{\mathscr{R}})$ and are called covering numbers of $\mathscr{R}$ and $\mathscr{R}$, respectively. The average value $\tau(\mathscr{R})=(1 / 2)(\tau(\underline{\mathscr{R}})+\tau(\overline{\mathscr{R}}))$ is called covering number of $\mathscr{R}$.

Definition 22. Let $\mathscr{R}=((\varphi A, \psi D),(\bar{\varphi} A, \bar{\psi} D))$ be a rough hypergraph on $Q$. The fractional covers of $\mathscr{R}$ and $\overline{\mathscr{R}}$ are, respectively, the mappings $\underline{f}: \underline{\varphi} A \longrightarrow \mathbb{R}^{+} \cup\{0\}$ and $\bar{f}: \bar{\varphi} A \longrightarrow \mathbb{R}^{+} \cup\{0\}$ such that

$$
\left.\begin{array}{l}
\sum_{k \in \underline{E}} \underline{f}(k) \quad \geq 1, \text { for each } \underline{E} \in \underline{\psi} D, \\
\sum_{k \in \bar{E}} \bar{f}(k) \quad \geq 1, \text { for each } \bar{E} \in \bar{\psi} D .
\end{array}\right]
$$

The value $\tau^{*}(\mathscr{R})=(1 / 2)\left(\min _{f} \sum_{k \in \varphi A} f \quad(k)+\min \right.$ $\sum_{k \in \bar{\varphi} A} \bar{f}(k)$ ) is called fractional covering number ( $\bar{f} \mathrm{C}$ number) of $\mathscr{R}$.

Definition 23. Let $\mathscr{R}=((\varphi A, \psi D),(\bar{\varphi} A, \bar{\psi} D))$ be a rough hypergraph on $Q$. The subsets $\underline{M} \subseteq \psi D$ and $\bar{M} \subseteq \bar{\psi} D$ are called matching if every pair of hyperedges from $\underline{M}$ and $\bar{M}$ are mutually disjoint. The cardinalities of maximal matchings are denoted by $v(\mathscr{R})$ and $\nu(\overline{\mathscr{R}})$ and are called matching numbers of $\mathscr{R}$ and $\mathscr{R}$, respectively. The matching number of $\mathscr{R}$ is computed as $\nu(\mathscr{R})=(1 / 2)(\nu(\underline{\mathscr{R}})+\nu(\overline{\mathscr{R}}))$

Definition 24. Let $\mathscr{R}=((\varphi A, \psi D),(\bar{\varphi} A, \bar{\psi} D))$ be a rough hypergraph on $Q$. The minimum number of mutually disjoint hyperedges whose union is the sets of vertices $\varphi A$ and $\bar{\varphi} A$ is called the partition number of $\mathscr{R}$ and $\overline{\mathscr{R}}$, respectively, denoted by $\rho(\underline{\mathscr{R}})$ and $\rho(\overline{\mathscr{R}})$. The value $\rho(\mathscr{R})=(1 / 2)(\rho(\underline{\mathscr{R}})+\rho(\overline{\mathscr{R}}))$ is called the partition number of $\mathscr{R}$. If such partitions do not exist, then $\rho(\mathscr{R})=\infty$.

We now study certain products of rough hypergraphs. In each product, the vertex set is the Cartesian product of the sets of vertices of all rough hypergraphs. The adjacency of edges is based on the adjacency properties defined in the product. Let $\mathscr{R}_{1} \circledast \mathscr{R}_{2}$ denote any product of two rough hypergraphs $\mathscr{R}_{1}$ and $\mathscr{R}_{2}$. For any rough hypergraph $\mathscr{R}$, if there exists another rough hypergraph $\mathscr{U}$ such that $\mathscr{R} \circledast \mathscr{U}=\mathscr{R}$, then $\mathscr{U}$ is called the unit element. Note that $\mathscr{U}$ must be a hypergraph with a single vertex and no loops.

Definition 25. A rough hypergraph $\mathscr{R}=(\underline{\mathscr{R}}, \overline{\mathscr{R}})$ is called conformal if $\mathscr{R}$ and $\overline{\mathscr{R}}$ are both conformal hypergraphs. That is, corresponding to each clique of 2 -section $[\mathscr{R}]_{2}$ (and $[\overline{\mathscr{R}}]_{2}$ ), there is a hyperedge in $\underline{\mathscr{R}}$ (and $\overline{\mathscr{R}}$ ).

Definition 26. Let $\mathscr{R}=(\mathscr{R}, \overline{\mathscr{R}})$ be a rough hypergraph. The collection of all hyperedges in $\underline{\mathscr{R}}$ (and $\overline{\mathscr{R}}$ ) containing a common vertex $k$ is called a star of $\mathscr{R}$ (and $\overline{\mathscr{R}}$ ), denoted by $\underline{\mathscr{R}}(k)$ (and $\overline{\mathscr{R}}(k))$. The pair $\mathscr{R}(k)=(\mathscr{R}(k), \overline{\mathscr{R}}(k))$ is called a rough star of $\mathscr{R}$. The subsets $\underline{E} \subseteq \psi D$ and $\underline{E} \subseteq \psi D$ are called intersecting families of $\mathscr{R}$ and $\overline{\mathscr{R}}$ if every pair of hyperedges of $\underline{E}$ and $\underline{E}$ have nonempty intersection. The pair $(\underline{E}, \underline{E})$ is called a rough intersecting family of $\mathscr{R}$. A rough hypergraph $\mathscr{R}$ is said to satisfy Helly property if each rough intersecting family in $\mathscr{R}$ is a rough star.

Definition 27. Let $\mathscr{R}_{1}^{\prime}$ and $\mathscr{R}_{2}^{\prime}$ be $p_{1}$-fold and $p_{2}$-fold coverings of rough hypergraphs $\mathscr{R}_{1}$ and $\mathscr{R}_{2}$ via covering projections $f_{1}=\left(f_{1}, f_{1}\right)$ and $f_{2}=\left(f_{2}, \bar{f}_{2}\right)$, respectively; then, the Cartesian product $(\underline{f}, \bar{f})=f=$ $f_{1} \square f_{2}=\left(\underline{f}_{1} \square \underline{f}_{2}, \bar{f}_{1} \square \bar{f}_{2}\right)$ is defined as

(1) $f\left(k_{1}, k_{2}\right)=\left(f_{1}\left(k_{1}\right), f_{2}\left(k_{2}\right)\right), \quad$ for all 
(1) Given a connected rough hypergraph $\mathscr{R}=((\varphi A, \psi D), \bar{\varphi} A, \bar{\psi} D)$

(2) Compute the L2-section $[\mathscr{R}]_{2}=\left(\left(\varphi A,[\psi D]_{2}\right),\left(\bar{\varphi} A,[\bar{\psi} D]_{2}\right)\right)$ of $\mathscr{R}$

(3) Using the Algorithm of Imrich and Peterin [52], decompose the labeled graphs $\left(\varphi A,[\psi D]_{2}\right)$ and $\left(\bar{\varphi} A\right.$, $\left.[\bar{\psi} D]_{2}\right)$ into prime factors w.r.t the Cartesian product. That is, the edges of $\left(\varphi A,[\psi D]_{2}\right)$ and $\left(\bar{\varphi} A,[\bar{\psi} D]_{2}\right)$ are colored w.r.t the copies of corresponding prime factors.

(4) Merge the factors if necessary

(5) Compute $[\mathscr{R}]_{2}^{-1}$ using the labeled and the PFD of $\mathscr{R}$ is obtained such that the colored copies are the prime factors

Algorithm 1: Method to compute PFD of a rough hypergraph.

(2) $\bar{f}\left(k_{1}, k_{2}\right)=\left(\bar{f}_{1}\left(k_{1}\right), \bar{f}_{2}\left(k_{2}\right)\right), \quad$ for $\left(k_{1}, k_{2}\right) \in \bar{\varphi} A_{1} \times \bar{\varphi} A_{2}$

(3) $f\left(\left\{k_{1}\right\} \times \underline{E}_{2}\right)=\underline{f}_{1}\left(k_{1}\right) \times \underline{f}_{2}\left(\underline{E}_{2}\right)$, for all $k_{1} \in \varphi A_{1}$, $\underline{\underline{E}}_{2} \in \underline{\phi} D_{2}$

(4) $\bar{f}\left(\left\{k_{1}\right\} \times \bar{E}_{2}\right)=\bar{f}_{1}\left(k_{1}\right) \times \bar{f}_{2}\left(\bar{E}_{2}\right)$, for all $k_{1} \in \bar{\varphi} A_{1}$, $\bar{E}_{2} \in \bar{\phi} D_{2}$

(5) $f\left(\underline{E}_{1} \times\left\{k_{2}\right\}\right)=\underline{f}_{1}\left(\underline{E}_{1}\right) \times \underline{f}_{2}\left(k_{2}\right)$, for all $\underline{E}_{1} \in \underline{\phi} D_{1}$, $\bar{k}_{1} \in \varphi A_{1}$

(6) $\bar{f}\left(\bar{E}_{1} \times\left\{k_{2}\right\}\right)=\bar{f}_{1}\left(\bar{E}_{1}\right) \times \bar{f}_{2}\left(k_{2}\right)$, for all $\bar{E}_{1} \in \bar{\phi} D_{1}$, $k_{1} \in \bar{\varphi} A_{1}$

Theorem 7. Let $\mathscr{R}^{\prime}{ }_{1}$ and $\mathscr{R}_{2}{ }_{2}$ be $p_{1}$-fold and $p_{2}$-fold coverings of rough hypergraphs $\mathscr{R}_{1}$ and $\mathscr{R}_{2}$ via covering projections $f_{1}$ and $f_{2}$, respectively; then, $\mathscr{R}_{1}{ }_{1} \square \mathscr{R}^{\prime}{ }_{2}$ is a $p_{1} p_{2}$-fold covering of $\mathscr{R}_{1} \square \mathscr{R}_{2}$ via covering projection $f_{1} \square f_{2}$.

Proof. The mapping $f=f_{1} \square f_{2}$ is given in Definition 27 . We first need to show that $f=(f, \bar{f})$ is a surjective homomorphism. Let $\left\{k_{1}^{\prime}\right\} \times \underline{E}_{2}{ }_{2}$ be a hyperedge in $\mathscr{R}^{\prime}{ }_{1} \square \mathscr{R}^{\prime}{ }_{2}$; then, $\underline{f}\left(\left\{k_{1}^{\prime}\right\} \times \underline{E}_{2}^{\prime}\right)=\underline{f}_{1}\left(k_{1}^{\prime}\right) \times \underline{f}_{2}\left(\underline{E}_{2}^{\prime}\right)$. Since $\underline{f}_{1}$ and $\underline{f}_{2}$ are homomorphisms, therefore $\underline{f}_{1}\left(k_{1}^{\prime}\right) \in \underline{\varphi} A_{2}^{\prime}$ and $\underline{f}_{2}\left(\underline{E}_{2}^{\prime}\right)$ is a hyperedge in $\underline{\mathscr{R}}_{2}$. Thus, $\underline{f}_{1}\left(\bar{k}_{1}^{\prime}\right) \times \underline{f}_{2}\left(\underline{E}_{2}^{\prime}\right)$ is a hyperedge in $\underline{\mathscr{R}}_{1} \square \mathscr{R}_{2}$. Similarly, $f\left(\underline{E}_{1}^{\prime} \times\left\{k_{2}^{\prime}\right\}\right)$ is a hyperedge $\underline{\mathscr{R}}_{1} \square \underline{\mathscr{R}}_{2}$ and $\bar{f}\left(\left\{k_{1}^{\prime}\right\} \times \bar{E}_{2}^{\prime}\right) ; \bar{f}\left(\bar{E}_{1}^{\prime} \times\left\{k_{2}^{\prime}\right\}\right)$ are hyperedges in $\overline{\mathscr{R}}_{1} \square \overline{\mathscr{R}}_{2}$ showing that $f$ is a homomorphism. The surjectivity of $\underline{f}_{1} \square f_{2}$ and $\bar{f}_{1} \square \bar{f}_{2}$ is obvious from the surjectivity of $\underline{f}_{1}, \underline{f}_{2}$, $\overline{\bar{f}}_{1}$, and $\bar{f}_{2}$.

Let $\left(k_{1}, k_{2}\right) \in \underline{\varphi} A_{1} \times \underline{\varphi} A_{2}$ be a vertex in $\underline{\mathscr{R}}_{1} \square \underline{\mathscr{R}}_{2}$; then,

$$
\begin{aligned}
\underline{f}_{1}^{-1}\left(k_{1}, k_{2}\right)= & \left\{\left(k_{1}^{\prime}, k_{2}^{\prime}\right) \mid \underline{f}_{1}\left(k_{1}^{\prime}\right)=k_{1}, \underline{f}_{2}\left(k_{2}^{\prime}\right)=k_{2}\right\} \\
\Rightarrow \underline{f}_{1}^{-1}\left(k_{1}, k_{2}\right)= & \underline{f}_{1}^{-1}\left(k_{1}\right) \times \underline{f}_{2}^{-1}\left(k_{2}\right) \\
& \Rightarrow\left|\underline{f}_{1}^{-1}\left(k_{1}, k_{2}\right)\right|=\left|\underline{f}_{1}^{-1}\left(k_{1}\right)\right| \times\left|\underline{f}_{2}^{-1}\left(k_{2}\right)\right|=p_{1} p_{2} .
\end{aligned}
$$

Similarly,

$$
\left|\bar{f}^{-1}\left(k_{1}, k_{2}\right)\right|=\left|\bar{f}_{1}^{-1}\left(k_{1}\right)\right| \times\left|\bar{f}_{2}^{-1}\left(k_{2}\right)\right|=p_{1} p_{2} .
$$

Consider a hyperedge $\left\{k_{1}\right\} \times \underline{E}_{2}$ in $\underline{\mathscr{R}}_{1} \square \mathscr{R}_{2}$; then,

$$
\begin{aligned}
\underline{f}^{-1}\left(\left\{k_{1}\right\} \times \underline{E}_{2}\right) & =\left\{\left(k_{1}^{\prime}, k_{2}^{\prime}\right) \mid \underline{f}_{1}\left(k_{1}^{\prime}\right)=k_{1}, \underline{f}_{2}\left(k_{2}^{\prime}\right) \in \underline{E}_{2}\right\} \\
& =\left\{\underline{f}_{1}^{-1}\left(k_{1}\right) \times \underline{f}_{2}^{-1}\left(k_{2}\right) \mid \underline{f}_{2}\left(k_{2}^{\prime}\right) \in \underline{E}_{2}\right\} \\
& =\underline{f}_{1}^{-1}\left(k_{1}\right) \times \underline{f}_{2}^{-1}\left(\underline{E}_{2}\right) \\
& \Rightarrow\left|\underline{f}^{-1}\left(\left\{k_{1}\right\} \times \underline{E}_{2}\right)\right|=\left|\underline{f}_{1}^{-1}\left(k_{1}\right)\right| \times\left|\underline{f}_{2}^{-1}\left(\underline{E}_{2}\right)\right|=p_{1} p_{2} .
\end{aligned}
$$

Similarly,

$$
\begin{aligned}
\left|\underline{f}_{1}^{-1}\left(\underline{E}_{1} \times\left\{k_{2}\right\}\right)\right|= & \left|\underline{f}_{1}^{-1}\left(\underline{E}_{1}\right)\right| \times\left|\underline{f}_{2}^{-1}\left(k_{2}\right)\right|=p_{1} p_{2} \\
& \cdot\left|\bar{f}^{-1}\left(\left\{k_{1}\right\} \times \bar{E}_{2}\right)\right|=\left|\bar{f}^{-1}\left(\bar{E}_{1} \times\left\{k_{2}\right\}\right)\right|=p_{1} p_{2} .
\end{aligned}
$$

Let $\left\{k_{1}\right\} \times \underline{E}_{2}$ be a hyperedge in $\underline{\mathscr{R}}_{1} \square \underline{\mathscr{R}}_{2}$ such that $\left\{k_{1}^{\prime}\right\} \times$ $\underline{E}_{2}^{\prime},\left\{k_{1}^{\prime}\right\} \times \underline{F}_{2}^{\prime} \in \underline{f}_{1}^{-1}\left(\left\{k_{1}\right\} \times \underline{E}_{2}\right) \quad$ and $\quad\left\{k_{1}^{\prime}\right\} \times \underline{E}_{2}^{\prime} \cap$ $\left\{k_{1}^{\prime}\right\} \times \underline{F}_{2}{ }_{2} \neq \varnothing$. It follows that $\underline{E}_{2}^{\prime} \cap \underline{F}^{\prime}{ }_{2} \neq \varnothing$, a contradiction. Similarly, we can prove the other cases. Hence, $f=(f, \bar{f})$ is covering projection and $\mathscr{R}^{\prime}{ }_{1} \square \mathscr{R}^{\prime}{ }_{2}$ is a $p_{1} p_{2}$-fold covering of $\mathscr{R}_{1} \square \mathscr{R}_{2}$.

Theorem 8. Let $\mathscr{R}_{1}$ and $\mathscr{R}_{2}$ be two rough hypergraphs; then, $\mathscr{R}_{1} \square \mathscr{R}_{2}$ is conformal if and only if $\mathscr{R}_{1}$ and $\mathscr{R}_{2}$ are conformal.

Proof. Let $\mathscr{R}_{1}$ and $\mathscr{R}_{2}$ be two conformal rough hypergraphs. Let $\mathscr{L}=(\underline{\mathscr{L}}, \overline{\mathscr{L}})$ be a rough clique in $\left[\mathscr{R}_{1} \square \mathscr{R}_{2}\right]_{2}$; then, by Lemma 7 , there are two possibilities.

Case 1. There exists a rough clique $\mathscr{L}_{1}=\left(\underline{\mathscr{L}}_{1}, \overline{\mathscr{L}}_{1}\right)$ in $\left[\mathscr{R}_{1}\right]_{2}$ such that $\underline{\mathscr{L}}=\mathscr{L}_{1} \times\left\{\underline{x}_{2}\right\}$, for some $\underline{x}_{2} \in \psi D_{2}$, and $\overline{\mathscr{L}}=\overline{\mathscr{L}}_{1} \times\left\{\bar{x}_{2}\right\}$, for some $\bar{x}_{2} \in \bar{\psi} D_{2}$. Since $\mathscr{L}$ is a rough clique, therefore, $\mathscr{L}_{1}$ is also a rough clique. As $\mathscr{R}_{1}$ is a conformal rough hypergraph, therefore there exist hyperedges $\underline{E}_{1}$ and $\bar{E}_{1}$ corresponding to $\mathscr{L}_{1}$ and $\overline{\mathscr{L}}_{1}$ in $\mathscr{R}_{1}$ and $\overline{\mathscr{R}}_{1}$. Thus, $\left(\underline{E}_{1} \times\left\{\underline{x}_{2}, \bar{E}_{1} \times\left\{\bar{x}_{2}\right)\right.\right.$ is a rough hyperedge corresponding to $\mathscr{L}_{1}$ showing that $\mathscr{R}_{1} \square \mathscr{R}_{2}$ is a conformal rough hypergraph.

Case 2. There exists a rough clique $\mathscr{L}_{2}=\left(\underline{\mathscr{L}}_{2}, \overline{\mathscr{L}}_{2}\right)$ in $\left[\mathscr{R}_{2}\right]_{2}$ such that $\mathscr{L}=\left\{\underline{x}_{1}\right\} \times \underline{\mathscr{L}}_{2}$, for some $\underline{x}_{1} \in \psi D_{1}$, and $\overline{\mathscr{L}}=\left\{\bar{x}_{1}\right\} \times \overline{\mathscr{L}}_{2}$, for some $\bar{x}_{1} \in \bar{\psi} D_{1}$. This case can be proved along the same lines as Case 1. Hence, $\mathscr{R}_{1} \square \mathscr{R}_{2}$ is a conformal rough hypergraph.

Conversely, let $\mathscr{R}_{1} \square \mathscr{R}_{2}$ be a conformal rough hypergraph. Let $\mathscr{L}_{1}$ and $\mathscr{L}_{2}$ be rough cliques in $\mathscr{R}_{1}$ and $\mathscr{R}_{2}$, respectively. By Lemma 7, $\mathscr{L}=\mathscr{L}_{1} \square \mathscr{L}_{2}$ is a rough clique in $\left[\mathscr{R}_{1} \square \mathscr{R}_{2}\right]_{2}$. Since $\mathscr{R}_{1} \square \mathscr{R}_{2}$ is a conformal rough hypergraph, 
therefore, there exists a rough hyperedge $E=(\underline{E}, \bar{E})$ in $\mathscr{R}_{1} \square \mathscr{R}_{2}$ corresponding to $\mathscr{L}$. By Lemma 7, there exist rough hyperedges $E_{1}$ and $E_{2}$ in $\mathscr{R}_{1}$ and $\mathscr{R}_{2}$ such that $E=E_{1} \square E_{2}$. Clearly, $E_{1}$ and $E_{2}$ correspond to in $\mathscr{L}_{1}$ and $\mathscr{L}_{2}$, respectively, proving that $\mathscr{R}_{1} \square \mathscr{R}_{2}$ are conformal rough hypergraphs.

Theorem 9. Let $\mathscr{R}_{1}$ and $\mathscr{R}_{2}$ be two rough hypergraphs; then, $\mathscr{R}_{1} \square \mathscr{R}_{2}$ has the Helly property iff $\mathscr{R}_{1}$ and $\mathscr{R}_{2}$ have the Helly property.

Definition 28. The dual of a rough hypergraph $\mathscr{R}=((\varphi A, \psi D),(\bar{\varphi} A, \bar{\psi} D))$ is a rough hypergraph $\mathscr{R}^{*}=\left(\left(\underline{\varphi} A^{*}, \psi D^{*}\right),\left(\bar{\varphi} A^{*}, \bar{\psi} D^{*}\right)\right)$, where

(1) The hyperedge set $\psi D$ of $\mathscr{R}$ is the vertex set of $\mathscr{R}^{*}$, that is, $\varphi A^{*}=\psi D=\left\{\underline{E}_{1}, \underline{E}_{2}, \ldots, \underline{E}_{r}\right\}$

(2) The edge set $\bar{\psi} D$ of $\mathscr{R}$ is the vertex set of $\mathscr{R}^{*}$, that is, $\bar{\varphi} A^{*}=\bar{\psi} D=\left\{\bar{E}_{1}, \bar{E}_{2}, \ldots, \bar{E}_{r}\right\}$

(3) If $|\varphi A|=m$, then $\psi D^{*}=\left\{\underline{K}_{1}, \underline{K}_{2}, \ldots, \underline{K}_{m}\right\}$ is the hyperedge set of $\mathscr{R}^{*}$ such that $\underline{K}_{i}=\left\{\underline{E}_{j} \mid k_{i} \in \underline{E}_{j}\right\}$, that is, $\underline{K}_{i}$ is the collection of those hyperedges of $\underline{\mathscr{R}}$ which share the common vertex $k_{i}$

(4) If $|\bar{\varphi} A|=n$, then $\bar{\psi} D^{*}=\left\{\bar{K}_{1}, \bar{K}_{2}, \ldots, \bar{K}_{n}\right\}$ is the hyperedge set of $\mathscr{R}^{*}$ such that $\bar{K}_{i}=\left\{\bar{E}_{j} \mid k_{i} \in \bar{E}_{j}\right\}$, that is, $\bar{K}_{i}$ is the collection of those hyperedges of $\overline{\mathscr{R}}$ which share the common vertices $k_{i}$

Remark 3. Let $\mathscr{R}_{1}$ and $\mathscr{R}_{2}$ be two rough hypergraphs; then, $\left(\mathscr{R}_{1} \square \mathscr{R}_{2}\right)^{*}$ may not be equal to $\mathscr{R}_{1}^{*} \square \mathscr{R}_{2}^{*}$. Since, for any two hypergraphs $H_{1}$ and $H_{2},\left(H_{1} \square H_{2}\right)^{*}$ is not equal to $h_{1}^{*} \square H_{2}^{*}$ in general, therefore, the equality also does not hold in the case of rough hypergraphs because a rough hypergraph contains two hypergraphs as upper and lower approximations. We discuss this fact using an example of two hypergraphs shown in Figures 7 and 8.

It is easy to check that $H_{1} \square H_{2}$ has nine edges and so $\left(H_{1} \square H_{2}\right)^{*}$ has nine vertices. But, $H_{1}^{*} \square H_{2}^{*}$ has three vertices. The vertex sets of $\left(H_{1} \square H_{2}\right)^{*}$ and $H_{1}^{*} \square H_{2}^{*}$ are not equal and it proves our claim.

3.2. Square Product. In this subsection, we introduce the concept of square product in rough hypergraphs and discuss its associativity, commutativity, distributivity, 2-section, rank, and antirank properties.

Definition 29. Let $\mathscr{R}_{1} * \mathscr{R}_{2} * \cdots * \mathscr{R}_{r}$ be any product of rough hypergraphs and $V\left(\mathscr{R}_{i}\right)$ denotes the vertex set of $\mathscr{R}_{i}$, for any $1 \leq i \leq r$. The mapping $p_{i}=\left(\underline{p}_{i}, \bar{p}_{i}\right): V\left(\mathscr{R}_{1} *\right.$ $\left.\mathscr{R}_{2} * \cdots * \mathscr{R}_{r}\right) \longrightarrow V\left(\mathscr{R}_{i}\right)$ is called the projection of $\mathscr{R}_{1} * \mathscr{R}_{2} * \cdots * \mathscr{R}_{r}$ onto ith factor $\mathscr{R}_{i}$, where $p_{i}: \mathscr{R}_{1} *$ $\underline{\mathscr{R}}_{2} * \cdots * \underline{\mathscr{R}}_{r} \longrightarrow \underline{\mathscr{R}}_{i}$ and $\bar{p}_{i}: \overline{\mathscr{R}}_{1} * \overline{\mathscr{R}}_{2} * \cdots * \overline{\mathscr{R}}_{r} \longrightarrow \overline{\mathscr{R}}_{i}$ are the projection mappings defined as

$$
\begin{array}{r}
\underline{p}_{i}\left(k_{1}, k_{2}, \ldots, k_{n}\right)=\bar{p}_{i}\left(k_{1}, k_{2}, \ldots, k_{n}\right)=k_{i}, \\
\qquad \text { for all } k_{i} \in Q_{i}, 1 \leq i \leq r .
\end{array}
$$
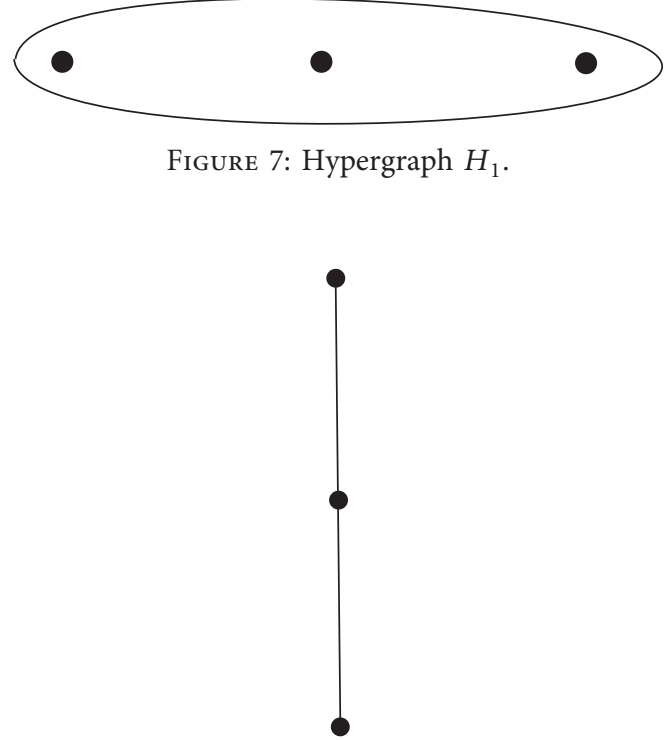

Figure 8: Hypergraph $H_{2}$.

Definition 30. Let $\mathscr{R}_{1}=\left(\mathscr{R}_{1}, \overline{\mathscr{R}}_{1}\right)$ and $\mathscr{R}_{2}=\left(\mathscr{R}_{2}, \overline{\mathscr{R}}_{2}\right)$ be two rough hypergraphs. The square product of $\mathscr{R}_{1}$ and $\mathscr{R}_{2}$ is a rough hypergraph $\mathscr{R}_{1} \times \mathscr{R}_{2}=\left(\underline{\mathscr{R}}_{1} \times \underline{\mathscr{R}}_{2}, \overline{\mathscr{R}}_{1} \times \overline{\mathscr{R}}_{2}\right)$ which is defined as

(1) $\underline{\mathscr{R}}_{1} \times \underline{\mathscr{R}}_{2}=\left(\underline{\varphi} A_{1} \times \underline{\varphi} A_{2}, \underline{\psi} D_{1} \times \underline{\psi} D_{2}\right)$

(a) $\varphi A_{1} \times \varphi A_{2}=\left\{\left(k_{1}, k_{2}\right) \mid k_{1} \in \varphi A_{1}, k_{2} \in \varphi A_{2}\right\}$

(b) $\underline{\psi} D_{1} \times \underline{\psi} D_{2}=\left\{\underline{E}_{1} \times \underline{E}_{2} \mid \underline{E}_{1} \in \underline{\psi} D_{1}, \underline{E}_{2} \in \underline{\psi} D_{2}\right\}$

(2) $\overline{\mathscr{R}}_{1} \square \overline{\mathscr{R}}_{2}=\left(\bar{\varphi} A_{1} \times \bar{\varphi} A_{2}, \bar{\psi} D_{1} \square \bar{\psi} D_{2}\right)$

(a) $\bar{\varphi} A_{1} \times \bar{\varphi} A_{2}=\left\{\left(k_{1}, k_{2}\right) \mid k_{1} \in \bar{\varphi} A_{1}, k_{2} \in \bar{\varphi} A_{2}\right\}$

(b) $\bar{\psi} D_{1} \times \bar{\psi} D_{2}=\left\{\bar{E}_{1} \times \bar{E}_{2} \mid \bar{E}_{1} \in \bar{\psi} D_{1}, \bar{E}_{2} \in \bar{\psi} D_{2}\right\}$

In short, $\underline{\mathscr{R}}_{1} \times \underline{\mathscr{R}}_{2}$ and $\overline{\mathscr{R}}_{1} \times \overline{\mathscr{R}}_{2}$ are the square products of lower approximate hypergraphs $\underline{\mathscr{R}}_{1}, \mathscr{R}_{2}$ and upper approximate hypergraphs $\overline{\mathscr{R}}_{1}, \overline{\mathscr{R}}_{2}$, respectively. Just like the square product of hypergraphs, the square product of rough hypergraphs is associative, distributive with respect to the disjoint union, commutative, and a unit $\mathcal{U}$ as a trivial hypergraph with a single vertex such that $\mathcal{U}=\overline{\mathcal{U}}$. That is, for any rough hypergraphs $\mathscr{R}, \mathscr{R}_{1}, \mathscr{R}_{2}$, and $\mathscr{R}_{3}$, the following properties hold:

(1) $\mathscr{R}_{1} \times\left(\mathscr{R}_{2} \times \mathscr{R}_{3}\right) \cong\left(\mathscr{R}_{1} \times \mathscr{R}_{2}\right) \times \mathscr{R}_{3}$

(2) $\mathscr{R}_{1} \times \mathscr{R}_{2} \cong \mathscr{R}_{2} \times \mathscr{R}_{1}$

(3) $\mathscr{R}_{1} \times\left(\mathscr{R}_{2} \cup \mathscr{R}_{3}\right) \cong\left(\mathscr{R}_{1} \times \mathscr{R}_{2}\right) \cup\left(\mathscr{R}_{1} \times \mathscr{R}_{2}\right)$

(4) $\mathscr{R} \times \mathscr{U} \cong \mathscr{R}$, where $\mathscr{U}$ is a single vertex hypergraph without loops

(5) The projections $p_{1}: V\left(\mathscr{R}_{1} \times \mathscr{R}_{2}\right) \longrightarrow\left(\mathscr{R}_{1}\right)$ and $p_{2}: V\left(\mathscr{R}_{1} \times \mathscr{R}_{2}\right) \longrightarrow V\left(\mathscr{R}_{2}\right)$ are homomorphisms

Definition 31. Let $\mathscr{R}$ be a rough hypergraph; then, $\mathscr{R}$ is called an $r$-uniform rough hypergraph if $\mathscr{R}$ and $\overline{\mathscr{R}}$ are both $r$-uniform hypergraphs; that is, for each $\underline{E} \in \psi D$ and $\bar{E} \in \bar{\psi} D,|\underline{E}|=|\bar{E}|=r$. 
Lemma 7. Let $\mathscr{R}_{1}$ and $\mathscr{R}_{2}$ be two r-uniform rough hypergraphs; then, $\left[\mathscr{R}_{1} \times \mathscr{R}_{2}\right]_{2} \cong\left[\mathscr{R}_{1}\right]_{2} \times\left[\mathscr{R}_{2}\right]_{2}$.

Proof. Since the vertex set of $\mathscr{R}_{i}$ and $\left[\mathscr{R}_{i}\right]_{2}$ is the same for $i=1,2$, therefore, the vertex set of $\left[\mathscr{R}_{1} \times \mathscr{R}_{2}\right]_{2}$ and $\left[\mathscr{R}_{1}\right]_{2} \times$ $\left[\mathscr{R}_{2}\right]_{2}$ is the same. It only needs to show that the set of hyperedges of $\left[\mathscr{R}_{1} \times \mathscr{R}_{2}\right]_{2}$ and $\left[\mathscr{R}_{1}\right]_{2} \times\left[\mathscr{R}_{2}\right]_{2}$ is the same. By Definition 30, $\left[\mathscr{R}_{1} \times \mathscr{R}_{2}\right]_{2}=\left(\left[\underline{\mathscr{R}}_{1} \times \underline{\mathscr{R}}_{2}\right]_{2},\left[\overline{\mathscr{R}}_{1} \times \overline{\mathscr{R}}_{2}\right]_{2}\right)$, where $\left[\underline{\mathscr{R}}_{1} \times \underline{\mathscr{R}}_{2}\right]_{2}=\left(\varphi A_{1} \times \varphi A_{2},\left[\psi \bar{D}_{1} \times \psi D_{2}\right]_{2}\right) \quad$ and $\left[\overline{\mathscr{R}}_{1} \times \overline{\mathscr{R}}_{2}\right]_{2}=\left(\bar{\varphi} A_{1} \times \bar{\varphi} \bar{A}_{2},\left[\bar{\psi} D_{1} \times \bar{\psi} \bar{D}_{2}\right]_{2}\right)$. As $\mathscr{R}_{1}$ and $\mathscr{R}_{2}$ are $r$-uniform rough hypergraphs, so we have

$$
\begin{aligned}
{\left[\underline{\psi} D_{1} \times \underline{\psi} D_{2}\right]_{2} } & =\left\{\left(x_{1}, x_{2}\right)\left(y_{1}, y_{2}\right) \mid\left\{\left(x_{1}, x_{2}\right),\left(y_{1}, y_{2}\right)\right\} \subseteq \underline{E} \in \underline{\psi} D_{1} \times \underline{\psi} D_{2}\right\} \\
& =\left\{\left(x_{1}, x_{2}\right)\left(x_{1}, y_{2}\right)\left|\left\{x_{1}, y_{1}\right\} \subseteq \underline{E}_{1} \in \underline{\psi} D_{1},\left\{x_{2}, y_{2}\right\} \subseteq \underline{E}_{2} \in \underline{\psi} D_{2},\right| \underline{E}_{1}|=| \underline{E}_{2} \mid\right\} \\
& =\left\{\left(x_{1}, x_{2}\right)\left(x_{1}, y_{2}\right) \mid x_{1} y_{1} \in\left[\underline{\psi} D_{1}\right]_{2}, x_{2} y_{2} \in\left[\underline{\psi} D_{2}\right]_{2}\right\} \\
& \Rightarrow\left[\underline{\psi} D_{1} \times \underline{\psi} D_{2}\right]_{2}=\left[\psi D_{1}\right]_{2} \times\left[\underline{\psi} D_{2}\right]_{2} .
\end{aligned}
$$

As the vertex set $\left[\underline{\mathscr{R}}_{1} \times \underline{\mathscr{R}}_{2}\right]_{2}$ and $\left[\underline{\mathscr{R}}_{1}\right]_{2} \times\left[\underline{\mathscr{R}}_{2}\right]_{2}$ is also the same, so $\left[\underline{\mathscr{R}}_{1} \times \underline{\mathscr{R}}_{2}\right]_{2} \cong\left[\underline{\mathscr{R}}_{1}\right]_{2} \times\left[\underline{\mathscr{R}}_{2}\right]_{2}$. Similarly, $\left[\overline{\mathscr{R}}_{1} \times \overline{\mathscr{R}}_{2}\right]_{2} \cong\left[\overline{\mathscr{R}}_{1}\right]_{2} \times\left[\overline{\mathscr{R}}_{2}\right]_{2}$. Hence, $\left[\mathscr{R}_{1} \times \mathscr{R}_{2}\right]_{2} \cong\left[\mathscr{R}_{1}\right]_{2}$ $\times\left[\mathscr{R}_{2}\right]_{2}$.

Theorem 10. Let $\mathscr{R}_{1}$ and $\mathscr{R}_{2}$ be two rough hypergraphs; then,

(1) $r\left(\mathscr{R}_{1} \times \mathscr{R}_{2}\right)=\max \left\{r\left(\mathscr{R}_{1}\right), r\left(\mathscr{R}_{2}\right),\left(r\left(\underline{\mathscr{R}}_{1}\right)+r\left(\overline{\mathscr{R}}_{2}\right)\right.\right.$ /2), $\left.\left(r\left(\underline{\mathscr{R}}_{2}\right)+r\left(\overline{\mathscr{R}}_{1}\right) / 2\right)\right\}$

(2) $s\left(\mathscr{R}_{1} \times \mathscr{R}_{2}\right)=\max \left\{s\left(\mathscr{R}_{1}\right), s\left(\mathscr{R}_{2}\right),\left(s\left(\underline{\mathscr{R}}_{1}\right)+\right.\right.$ $\left.\left.s\left(\overline{\mathscr{R}}_{2}\right) / 2\right),\left(s\left(\underline{\mathscr{R}}_{2}\right)+s\left(\overline{\mathscr{R}}_{1}\right) / 2\right)\right\}$
Proof. Since, by Definition 30, $\mathscr{R}_{1} \times \mathscr{R}_{2}=$ $\left(\underline{\mathscr{R}}_{1} \times \underline{\mathscr{R}}_{2}, \overline{\mathscr{R}}_{1} \times \overline{\mathscr{R}}_{2}\right)$, we first need to compute $r\left(\underline{\mathscr{R}}_{1} \times \underline{\mathscr{R}}_{2}\right)$ and $r\left(\overline{\mathscr{R}}_{1} \times \overline{\mathscr{R}}_{2}\right)$. By Definition 6,

$$
\begin{aligned}
& r\left(\underline{\mathscr{R}}_{1} \times \underline{\mathscr{R}}_{2}\right)=\max _{\underline{E} \in \psi D_{1} \times \psi D_{2}}|\underline{E}| \\
& =\max \left\{\underline{E} \mid p_{1}(\underline{E})=\underline{E}_{1} \in \underline{\psi} D_{1}, p_{2}(\underline{E})=\underline{E}_{2} \in \underline{\psi} D_{2}\right\} \\
& =\max \left\{\max _{\underline{E}_{1} \in \underline{\psi} D_{1}}\left|\underline{E}_{1}\right|, \max _{\underline{E}_{2} \in \underline{\psi} D_{2}}\left|\underline{E}_{2}\right|\right\} \\
& \Rightarrow r\left(\underline{\mathscr{R}}_{1} \times \underline{\mathscr{R}}_{2}\right)=\max \left\{r\left(\underline{\mathscr{R}}_{1}\right), r\left(\underline{\mathscr{R}}_{2}\right)\right\} \text {. }
\end{aligned}
$$

Similarly, $r\left(\overline{\mathscr{R}}_{1} \times \overline{\mathscr{R}}_{2}\right)=\max \left\{r\left(\overline{\mathscr{R}}_{1}\right), r\left(\overline{\mathscr{R}}_{2}\right)\right\}$. Hence,

$$
\begin{aligned}
r\left(\mathscr{R}_{1} \times \mathscr{R}_{2}\right) & =\frac{1}{2}\left\{\max \left\{r\left(\underline{\mathscr{R}}_{1}\right), r\left(\underline{\mathscr{R}}_{2}\right)\right\}+\max r\left(\overline{\mathscr{R}}_{1}\right), r\left(\overline{\mathscr{R}}_{2}\right)\right\} \\
& =\max \left\{\frac{r\left(\underline{\mathscr{R}}_{1}\right)+r\left(\overline{\mathscr{R}}_{1}\right)}{2}, \frac{r\left(\underline{\mathscr{R}}_{2}\right)+r\left(\overline{\mathscr{R}}_{2}\right)}{2}, \frac{r(\underline{\mathscr{R}})+r\left(\overline{\mathscr{R}}_{2}\right)}{2}, \frac{r\left(\underline{\mathscr{R}}_{2}\right)+r\left(\overline{\mathscr{R}}_{1}\right)}{2}\right\} \\
& \Rightarrow r\left(\underline{\mathscr{R}}_{1} \times \underline{\mathscr{R}}_{2}\right)=\max \left\{r\left(\mathscr{R}_{1}\right), r\left(\mathscr{R}_{2}\right), \frac{r\left(\frac{\mathscr{R}}{1}\right)+r\left(\overline{\mathscr{R}}_{2}\right)}{2}, \frac{r\left(\underline{\mathscr{R}}_{2}\right)+r\left(\overline{\mathscr{R}}_{1}\right)}{2}\right\} .
\end{aligned}
$$

Using Definition 6, the antirank of $\mathscr{R}_{1} \times \mathscr{R}_{2}$ is given as

$$
\begin{aligned}
s\left(\underline{\mathscr{R}}_{1} \times \underline{\mathscr{R}}_{2}\right) & =\min _{\underline{E} \in \underline{\psi} D_{1} \times \underline{\underline{D_{2}}}}|\underline{E}| \\
& =\min \left\{|\underline{E}| \mid p_{1}(\underline{E})=\underline{E}_{1} \in \underline{\psi} D_{1}, p_{2}(\underline{E})=\underline{E}_{2} \in \underline{\psi} D_{2}\right\} \\
& =\max \left\{\min _{\underline{E}_{1} \in \underline{\underline{D_{1}}}}\left|\underline{E}_{1}\right|, \min _{\underline{E}_{2} \in \underline{\psi} D_{2}}\left|\underline{E}_{2}\right|\right\} \\
& \Rightarrow s\left(\underline{\mathscr{R}}_{1} \times \underline{\mathscr{R}}_{2}\right)=\max \left\{s\left(\underline{\mathscr{R}}_{1}\right), s\left(\underline{\mathscr{R}}_{2}\right)\right\} .
\end{aligned}
$$


Similarly, $s\left(\overline{\mathscr{R}}_{1} \times \overline{\mathscr{R}}_{2}\right)=\max \left\{s\left(\overline{\mathscr{R}}_{1}\right), s\left(\overline{\mathscr{R}}_{2}\right)\right\}$. Hence,

$$
\begin{aligned}
s\left(\mathscr{R}_{1} \times \mathscr{R}_{2}\right) & =\frac{1}{2}\left\{\max \left\{s\left(\underline{\mathscr{R}}_{1}\right), s\left(\underline{\mathscr{R}}_{2}\right)\right\}+\max s\left(\overline{\mathscr{R}}_{1}\right), s\left(\overline{\mathscr{R}}_{2}\right)\right\} \\
& =\max \left\{\frac{s\left(\underline{\mathscr{R}}_{1}\right)+s\left(\overline{\mathscr{R}}_{1}\right)}{2}, \frac{s\left(\underline{\mathscr{R}}_{2}\right)+s\left(\overline{\mathscr{R}}_{2}\right)}{2}, \frac{s\left(\underline{\mathscr{R}}_{1}\right)+s\left(\overline{\mathscr{R}}_{2}\right)}{2}, \frac{s\left(\underline{\mathscr{R}}_{2}\right)+s\left(\overline{\mathscr{R}}_{1}\right)}{2}\right\} \\
\Rightarrow s\left(\mathscr{R}_{1} \times \mathscr{R}_{2}\right) & =\max \left\{s\left(\mathscr{R}_{1}\right), s\left(\mathscr{R}_{2}\right), \frac{s\left(\underline{\mathscr{R}}_{1}\right)+s\left(\overline{\mathscr{R}}_{2}\right)}{2}, \frac{s\left(\underline{\mathscr{R}}_{2}\right)+s\left(\overline{\mathscr{R}}_{1}\right)}{2}\right\} .
\end{aligned}
$$

3.3. Direct Product. In this section, we introduce the extension of the concept of the square product to direct product of rough hypergraphs and discuss its associativity, commutativity, distributivity, 2-section, rank, and antirank properties.

Definition 32. Let $\mathscr{R}_{1}=\left(\underline{\mathscr{R}}_{1}, \overline{\mathscr{R}}_{1}\right)$ and $\mathscr{R}_{2}=\left(\underline{\mathscr{R}}_{2}, \overline{\mathscr{R}}_{2}\right)$ be two rough hypergraphs. The direct product $\times$ of $\mathscr{R}_{1}$ and $\mathscr{R}_{2}$ is a rough hypergraph $\mathscr{R}_{1} \times \mathscr{R}_{2}=\left(\underline{\mathscr{R}}_{1} \times \underline{\mathscr{R}}_{2}, \overline{\mathscr{R}}_{1} \times \overline{\mathscr{R}}_{2}\right)$ which is defined as

(1) $\underline{\mathscr{R}}_{1} \overline{\widetilde{R}} \underline{\mathscr{R}}_{2}=\left(\underline{\varphi} A_{1} \times \underline{\varphi} A_{2}, \underline{\psi} D_{1} \overline{\times} \underline{\psi} D_{2}\right)$

(a) $\varphi A_{1} \times \varphi A_{2}=\left\{\left(k_{1}, k_{2}\right) \mid k_{1} \in \varphi A_{1}, k_{2} \in \varphi A_{2}\right\}$

(b) $\bar{\psi} D_{1} \times \bar{\psi} D_{2}=\left\{\underline{E} \mid p_{1} \quad(\underline{E}) \in \psi D_{1}, p_{2}(\underline{E}) \subseteq \underline{E}_{2}\right.$

$\left.\epsilon \underline{\psi} D_{2}\right\} \cup\left\{\underline{E} \mid p_{1}(\underline{E}) \subseteq \underline{E}_{1} \in \underline{\psi} D_{1}^{-}, p_{2}(\underline{E}) \in \underline{\psi} D_{2}\right\}$

(2) $\overline{\mathscr{R}}_{1} \overline{\times} \overline{\mathscr{R}}_{2}=\left(\bar{\varphi} A_{1} \times \bar{\varphi} A_{2}, \bar{\psi} D_{1} \overline{\times} \bar{\psi} D_{2}\right)$

(a) $\bar{\varphi} A_{1} \times \bar{\varphi} A_{2}=\left\{\left(k_{1}, k_{2}\right) \mid k_{1} \in \bar{\varphi} A_{1}, k_{2} \in \bar{\varphi} A_{2}\right\}$

(b) $\bar{\psi} D_{1} \times \bar{\psi} D_{2}=\left\{\bar{E} \mid p_{1}(\bar{E}) \in \bar{\psi} D_{1}, p_{2}\right.$

$\left.(\bar{E}) \subseteq \bar{E}_{2} \bar{\psi} D_{2}\right\} \cup\left\{\bar{E} \mid p_{1}(\bar{E}) \subseteq \bar{E}_{1} \in\right.$

$\left.\bar{\psi} D_{1}, p_{2}(\bar{E}) \in \bar{\psi} D_{2}\right\}$

In short, $\underline{\mathscr{R}}_{1} \times \underline{\mathscr{R}}_{2}$ and $\overline{\mathscr{R}}_{1} \overline{\times} \overline{\mathscr{R}}_{2}$ are the MRP direct products of lower approximate hypergraphs $\underline{\mathscr{R}}_{1}, \mathscr{R}_{2}$ and upper approximate hypergraphs $\overline{\mathscr{R}}_{1}, \overline{\mathscr{R}}_{2}$, respectively. Just like the MRP direct product of hypergraphs, the MRP direct product of rough hypergraphs is associative, right distributive with respect to the disjoint union, commutative, and a unit $\mathcal{U}$ as a trivial hypergraph with a single vertex such that $\underline{\mathscr{U}}=\overline{\mathscr{U}}$. That is, for any rough hypergraphs $\mathscr{R}, \mathscr{R}_{1}, \mathscr{R}_{2}$, and $\overline{\mathscr{R}}_{3}$, the following properties hold:

(1) $\mathscr{R}_{1} \overline{\times}\left(\mathscr{R}_{2} \overline{\times} \mathscr{R}_{3}\right) \cong\left(\mathscr{R}_{1} \overline{\times} \mathscr{R}_{2}\right) \overline{\times} \mathscr{R}_{3}$

(2) $\mathscr{R}_{1} \overline{\times} \mathscr{R}_{2} \cong \mathscr{R}_{2} \times \mathscr{R}_{1}$

(3) $\mathscr{R}_{1} \overline{\times}\left(\mathscr{R}_{2} \cup \mathscr{R}_{3}\right) \cong\left(\mathscr{R}_{1} \overline{\times} \mathscr{R}_{2}\right) \cup\left(\mathscr{R}_{1} \times \mathscr{R}_{2}\right)$

(4) $\mathscr{R} \times \mathscr{U} \cong \mathscr{R}$, where $\mathcal{U}$ is a single vertex hypergraph without loops

(5) The projections $p_{1}: V\left(\mathscr{R}_{1} \overline{\times} \mathscr{R}_{2}\right) \longrightarrow\left(\mathscr{R}_{1}\right)$ and $p_{2}: V\left(\mathscr{R}_{1} \overline{\times} \mathscr{R}_{2}\right) \longrightarrow V\left(\mathscr{R}_{2}\right)$ may not be weak homomorphisms

Lemma 8. Let $\mathscr{R}_{1}$ and $\mathscr{R}_{2}$ be two rough hypergraphs; then, $\left[\mathscr{R}_{1} \times \mathscr{R}_{2}\right]_{2} \cong\left(\left[\mathscr{R}_{1}\right]_{2} \square\left[\mathscr{R}_{2}\right]_{2}\right) \cup\left(\left[\mathscr{R}_{1}\right]_{2} \times\left[\mathscr{R}_{2}\right]_{2}\right)$.

Proof. Since the vertex set of $\mathscr{R}_{i}$ and $\left[\mathscr{R}_{i}\right]_{2}$ is the same for $i=1,2$, therefore, the vertex set of $\left[\mathscr{R}_{1} \overline{\times} \mathscr{R}_{2}\right]_{2}$ and the union of $\left[\mathscr{R}_{1}\right]_{2} \square\left[\mathscr{R}_{2}\right]_{2}$ and $\left[\mathscr{R}_{1}\right]_{2} \times\left[\mathscr{R}_{2}\right]_{2}$ is the same. It only needs to show that the set of hyperedges of $\left[\mathscr{R}_{1} \times \mathscr{R}_{2}\right]_{2}$ and $\left(\left[\mathscr{R}_{1}\right]_{2} \square\left[\mathscr{R}_{2}\right]_{2}\right) \cup\left(\left[\mathscr{R}_{1}\right]_{2} \times\left[\mathscr{R}_{2}\right]_{2}\right)$ is the same. By Definition 32, $\left[\mathscr{R}_{1} \times \mathscr{R}_{2}\right]_{2}=\left(\left[\underline{\mathscr{R}}_{1} \times \underline{\mathscr{R}}_{2}\right]_{2},\left[\overline{\mathscr{R}}_{1} \times \overline{\mathscr{R}}_{2}\right]_{2}\right)$, where $\left[\underline{\mathscr{R}}_{1} \times \underline{\mathscr{R}}_{2}\right]_{2}=\left(\underline{\varphi} A_{1} \times \underline{\varphi} A_{2},\left[\psi D_{1} \times \bar{\psi} D_{2}\right]_{2}\right)$ and $\left[\overline{\mathscr{R}}_{1} \times \overline{\mathscr{R}}_{2}\right]_{2}$ $=\left(\bar{\varphi} A_{1} \times \bar{\varphi} A_{2},\left[\bar{\psi} D_{1} \times \bar{\psi} D_{2}\right]_{2}\right)$. So, we have

$$
\begin{aligned}
& {\left[\underline{\psi} D_{1} \times \underline{\psi} D_{2}\right]_{2}=\left\{\left(x_{1}, x_{2}\right)\left(y_{1}, y_{2}\right) \mid\left\{\left(x_{1}, x_{2}\right),\left(y_{1}, y_{2}\right)\right\} \subseteq \underline{E} \in \underline{\psi} D_{1} \times \underline{\psi} D_{2}\right\}} \\
& {\left[\psi D_{1} \overline{\times} \underline{\psi} D_{2}\right]_{2}=\left\{\left(x_{1}, x_{2}\right)\left(y_{1}, y_{2}\right) \mid\left\{\left(x_{1}, x_{2}\right),\left(y_{1}, y_{2}\right)\right\} \subseteq \underline{E} \in \underline{\psi} D_{1} \times \underline{\psi} D_{2}\right\}} \\
& =\left\{\left(x_{1}, x_{2}\right)\left(y_{1}, y_{2}\right) \mid x_{1}=y_{1},\left\{x_{2}, y_{2}\right\} \subseteq p_{2}(\underline{E}) \in \underline{\psi} D_{2}\right\} \\
& \cup\left\{\left(x_{1}, x_{2}\right)\left(x_{1}, y_{2}\right) \mid\left\{x_{1}, y_{1}\right\} \subseteq p_{1}(\underline{E}) \in \underline{\psi} D_{1}, x_{2}=y_{2}\right\} \\
& \cup\left\{\left(x_{1}, x_{2}\right)\left(x_{1}, y_{2}\right)\left|\left\{x_{1}, y_{1}\right\} \subseteq p_{1}(\underline{E}) \in \underline{\psi D_{1}},\left\{x_{2}, y_{2}\right\} \subseteq p_{2}(\underline{E}) \in \underline{\psi D_{2}},\right| \underline{E}_{1}|=| \underline{E}_{2} \mid\right\} \\
& =\left\{\left(x_{1}, x_{2}\right)\left(x_{1}, y_{2}\right) \mid x_{1}, y_{1} \in \underline{\varphi} A_{1}, x_{2} y_{2} \in\left[\underline{\psi} D_{2}\right]_{2}\right\} \\
& \left\{\left(x_{1}, x_{2}\right)\left(x_{1}, y_{2}\right) \mid x_{1} y_{1} \in\left[\underline{\psi} D_{1}\right]_{2}, x_{2}, y_{2} \in \underline{\varphi} A_{2}\right\} \\
& \left\{\left(x_{1}, x_{2}\right)\left(x_{1}, y_{2}\right) \mid x_{1} y_{1} \in\left[\underline{\psi} D_{1}\right]_{2}, x_{2} y_{2} \in\left[\underline{\psi} D_{2}\right]_{2}\right\} \\
& \Rightarrow\left[\psi D_{1} \overline{\times} \quad \underline{\psi} D_{2}\right]_{2}=\left(\left[\underline{\psi} D_{1}\right]_{2} \square\left[\psi D_{2}\right]_{2}\right) \cup\left(\left[\underline{\psi} D_{1}\right]_{2} \times\left[\underline{\psi} D_{2}\right]_{2}\right) .
\end{aligned}
$$


Thus, $\left[\underline{\mathscr{R}}_{1} \times \breve{\mathscr{R}}_{2}\right]_{2} \cong\left(\left[\underline{\mathscr{R}_{1}}\right]_{2} \square\left[\underline{\mathscr{R}}_{2}\right]_{2}\right) \cup\left(\left[\underline{\mathscr{R}}_{1}\right]_{2} \times\left[\underline{\mathscr{R}}_{2}\right]_{2}\right)$. Similarly, $\left[\overline{\mathscr{R}}_{1} \times \overline{\mathscr{R}}_{2}\right]_{2} \cong\left(\left[\overline{\mathscr{R}}_{1}\right]_{2} \square\left[\overline{\mathscr{R}}_{2}\right]_{2}\right) \cup\left(\left[\overline{\mathscr{R}}_{1}\right]_{2} \times\left[\overline{\mathscr{R}}_{2}\right]_{2}\right)$. Hence,

$\left[\mathscr{R}_{1} \times \mathscr{R}_{2}\right]_{2} \cong\left(\left[\mathscr{R}_{1}\right]_{2} \square\left[\mathscr{R}_{2}\right]_{2}\right) \cup\left(\left[\mathscr{R}_{1}\right]_{2} \times\left[\mathscr{R}_{2}\right]_{2}\right)$.

Theorem 11. Let $\mathscr{R}_{1}$ and $\mathscr{R}_{2}$ be two rough hypergraphs; then,

(1) $r\left(\mathscr{R}_{1} \times \mathscr{R}_{2}\right)=\max \left\{r\left(\mathscr{R}_{1}\right), r\left(\mathscr{R}_{2}\right)\right.$, $\left.\left(r\left(\mathscr{R}_{1}\right)+r\left(\overline{\mathscr{R}}_{2}\right) / 2\right),\left(r\left(\underline{\mathscr{R}}_{2}\right)+r\left(\overline{\mathscr{R}}_{1}\right) / 2\right)\right\}$

(2) $s\left(\mathscr{R}_{1} \times \mathscr{R}_{2}\right)=\min \left\{s\left(\mathscr{R}_{1}\right), s\left(\mathscr{R}_{2}\right),\left(s\left(\mathscr{R}_{1}\right)+\right.\right.$ $\left.\left.s\left(\overline{\mathscr{R}}_{2}\right) / 2\right),\left(s\left(\mathscr{R}_{2}\right)+s\left(\overline{\mathscr{R}}_{1}\right) / 2\right)\right\}$
Proof. Since, by Definition 32, $\mathscr{R}_{1} \times \mathscr{R}_{2}=$ $\left(\underline{\mathscr{R}}_{1} \times \underline{\mathscr{R}}_{2}, \overline{\mathscr{R}}_{1} \times \overline{\mathscr{R}}_{2}\right)$, we first need to compute $r\left(\underline{\mathscr{R}}_{1} \times \mathscr{\mathscr { R }}_{2}\right)$ and $r\left(\mathscr{\mathscr { R }}_{1} \times \mathscr{R}_{2}\right)$. By Definition 6,

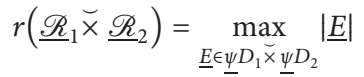

$$
\begin{aligned}
& =\max \left\{|\underline{E}| \mid p_{1}(\underline{E})=\underline{E}_{1} \in \psi D_{1}, p_{2}(\underline{E}) \subseteq \underline{E}_{2} \in \psi D_{2}\right\} \\
& \cup\left\{|\underline{E}| \mid p_{1}(\underline{E}) \subseteq \underline{E}_{1} \in \underline{\psi} D_{1}, p_{2}(\underline{E})=\underline{E}_{2} \in \underline{\psi} D_{2}\right\} \\
& =\max \left\{\max _{\underline{E}_{1} \in \psi D_{1}}\left|\underline{E}_{1}\right|, \max _{\underline{E}_{2} \in \psi D_{2}}\left|\underline{E}_{2}\right|\right\} \\
& \Rightarrow r\left(\mathscr{R}_{1} \times \mathscr{\mathscr { R }}_{2}\right)=\max r\left(\underline{\mathscr{R}}_{1}\right), r\left(\underline{\mathscr{R}}_{2}\right) \text {. }
\end{aligned}
$$

Similarly, $r\left(\overline{\mathscr{R}}_{1} \times \overline{\mathscr{R}}_{2}\right)=\max \left\{r\left(\overline{\mathscr{R}}_{1}\right), r\left(\overline{\mathscr{R}}_{2}\right)\right\}$. Hence,

$$
\begin{aligned}
r\left(\mathscr{R}_{1} \times \mathscr{R}_{2}\right) & =\frac{1}{2}\left\{\max \left\{r\left(\underline{\mathscr{R}}_{1}\right), r\left(\underline{\mathscr{R}}_{2}\right)\right\}+\max r\left(\overline{\mathscr{R}}_{1}\right), r\left(\overline{\mathscr{R}}_{2}\right)\right\} \\
& =\max \left\{\frac{r\left(\underline{\mathscr{R}}_{1}\right)+r\left(\overline{\mathscr{R}}_{1}\right)}{2}, \frac{r\left(\underline{\mathscr{R}}_{2}\right)+r\left(\overline{\mathscr{R}}_{2}\right)}{2}, \frac{r\left(\underline{\mathscr{R}}_{1}\right)+r\left(\overline{\mathscr{R}}_{2}\right)}{2}, \frac{r\left(\mathscr{\mathscr { R }}_{2}\right)+r\left(\overline{\mathscr{R}}_{1}\right)}{2}\right\} \\
& \Rightarrow r\left(\mathscr{R}_{1} \overline{\times} \mathscr{R}_{2}\right)=\max \left\{r\left(\mathscr{R}_{1}\right), r\left(\mathscr{R}_{2}\right), \frac{r\left(\underline{\mathscr{R}}_{1}\right)+r\left(\overline{\mathscr{R}}_{2}\right)}{2}, \frac{r\left(\mathscr{\mathscr { R }}_{2}\right)+r\left(\overline{\mathscr{R}}_{1}\right)}{2}\right\} .
\end{aligned}
$$

Using Definition 6, the antirank of $\mathscr{R}_{1} \times \mathscr{R}_{2}$ is given as

$$
\begin{aligned}
& s\left(\underline{\mathscr{R}}_{1} \times \underline{\mathscr{R}}_{2}\right)=\min _{\underline{E} \in \psi D_{1} \times \psi D_{2}}|\underline{E}| \\
& =\min \left\{|\underline{E}| \mid p_{1}(\underline{E})=\underline{E}_{1} \in \psi D_{1}, p_{2}(\underline{E}) \subseteq \underline{E}_{2} \in \psi D_{2}\right\} \\
& \cup\left\{|\underline{E}| \mid p_{1}(\underline{E}) \subseteq \underline{E}_{1} \in \underline{\psi} D_{1}, p_{2}(\underline{E})=\underline{E}_{2} \in \underline{\psi} D_{2}\right\} \\
& =\min \left\{\min _{\underline{E}_{1} \in \underline{\psi} D_{1}}\left|\underline{E}_{1}\right|, \min _{\underline{E}_{2} \in \underline{\psi} D_{2}}\left|\underline{E}_{2}\right|\right\} \\
& \Rightarrow s\left(\underline{\mathscr{R}}_{1} \times \underline{\mathscr{R}}_{2}\right)=\min \left\{s\left(\underline{\mathscr{R}}_{1}\right), s\left(\underline{\mathscr{R}}_{2}\right)\right\} \text {. }
\end{aligned}
$$

Similarly, $s\left(\overline{\mathscr{R}}_{1} \overline{\times} \overline{\mathscr{R}}_{2}\right)=\max \left\{s\left(\overline{\mathscr{R}}_{1}\right), s\left(\overline{\mathscr{R}}_{2}\right)\right\}$. Hence,

$$
\begin{aligned}
s\left(\mathscr{R}_{1} \times \mathscr{R}_{2}\right) & =\frac{1}{2}\left\{\min \left\{s\left(\underline{\mathscr{R}}_{1}\right), s\left(\underline{\mathscr{R}}_{2}\right)\right\}+\min s\left(\overline{\mathscr{R}}_{1}\right), s\left(\overline{\mathscr{R}}_{2}\right)\right\} \\
& =\min \left\{\frac{s\left(\underline{\mathscr{R}}_{1}\right)+s\left(\overline{\mathscr{R}}_{1}\right)}{2}, \frac{s\left(\underline{\mathscr{R}}_{2}\right)+s\left(\overline{\mathscr{R}}_{2}\right)}{2}, \frac{s\left(\underline{\mathscr{R}}_{1}\right)+s\left(\overline{\mathscr{R}}_{2}\right)}{2}, \frac{s\left(\mathscr{\mathscr { R }}_{2}\right)+s\left(\overline{\mathscr{R}}_{1}\right)}{2}\right\} \\
& \Rightarrow s\left(\mathscr{R}_{1} \overline{\times} \mathscr{R}_{2}\right)=\min \left\{s\left(\mathscr{R}_{1}\right), s\left(\mathscr{R}_{2}\right), \frac{s\left(\underline{\mathscr{R}}_{1}\right)+s\left(\overline{\mathscr{R}}_{2}\right)}{2}, \frac{s\left(\mathscr{R}_{2}\right)+s\left(\overline{\mathscr{R}}_{1}\right)}{2}\right\} .
\end{aligned}
$$


3.4. Union and Intersection. In this subsection, we introduce the concepts union of the intersection of rough hypergraphs and study their properties.

Definition 33. Let $\mathscr{R}_{1}=\left(\underline{\mathscr{R}}_{1}, \overline{\mathscr{R}}_{1}\right)$ and $\mathscr{R}_{2}=\left(\underline{\mathscr{R}}_{2}, \overline{\mathscr{R}}_{2}\right)$ be two rough hypergraphs. The union $\mathrm{f} \mathscr{R}_{1}$ and $\mathscr{R}_{2}$ is a rough hypergraph $\quad \mathscr{R}_{1} \cup \mathscr{R}_{2}=\left(\underline{\mathscr{R}}_{1} \cup \underline{\mathscr{R}}_{2}, \overline{\mathscr{R}}_{1} \cup \overline{\mathscr{R}}_{2}\right)$, where $\underline{\mathscr{R}}_{1} \cup \underline{\mathscr{R}}_{2}=\left(\varphi A_{1} \cup \varphi A_{2}, \psi D_{1} \cup \psi D_{2}\right) \quad$ and $\quad \overline{\mathscr{R}}_{1} \cup \overline{\mathscr{R}}_{2}=$ $\left(\bar{\varphi} A_{1} \cup \bar{\varphi} A_{2}, \overline{\bar{\psi}} D_{1} \cup \overline{\bar{\psi}} D_{2}\right)$.

The intersection $f \mathscr{R}_{1}$ and $\mathscr{R}_{2}$ is a rough hypergraph $\mathscr{R}_{1} \cap \mathscr{R}_{2}=\left(\underline{\mathscr{R}}_{1} \cap \underline{\mathscr{R}}_{2}, \overline{\mathscr{R}}_{1} \cap \overline{\mathscr{R}}_{2}\right)$, where $\quad \underline{\mathscr{R}}_{1} \cap \underline{\mathscr{R}}_{2}=$ $\left(\varphi A_{1} \cap \varphi A_{2}, \psi D_{1} \cap \psi D_{2}\right)$ and $\overline{\mathscr{R}}_{1} \cap \overline{\mathscr{R}}_{2}=\left(\bar{\varphi} A_{1} \cap \bar{\varphi} A_{2}\right.$, $\left.\bar{\psi} \bar{D}_{1} \cap \bar{\psi} \bar{D}_{2}\right)$.

In short, $\underline{\mathscr{R}}_{1} \cup \underline{\mathscr{R}}_{2}$ and $\underline{\mathscr{R}}_{1} \cap \underline{\mathscr{R}}_{2}$ are the union and intersection of lower approximate hypergraphs $\underline{\mathscr{R}}_{1}$ and $\underline{R}_{2}$. Similarly, for the upper approximate hypergraphs, just like the union (intersection) of hypergraphs, the union (intersection) of rough hypergraphs is associative, commutative, and distributive. That is, for any rough hypergraphs $\mathscr{R}_{1}, \mathscr{R}_{2}$, and $\mathscr{R}_{3}$, the following properties hold:

(1) $\mathscr{R}_{1} \cup\left(\mathscr{R}_{2} \cup \mathscr{R}_{3}\right) \cong\left(\mathscr{R}_{1} \cup \mathscr{R}_{2}\right) \cup \mathscr{R}_{3}$

(2) $\mathscr{R}_{1} \cap\left(\mathscr{R}_{2} \cap \mathscr{R}_{3}\right) \cong\left(\mathscr{R}_{1} \cap \mathscr{R}_{2}\right) \cap \mathscr{R}_{3}$

(3) $\mathscr{R}_{1} \cup \mathscr{R}_{2} \cong \mathscr{R}_{2} \cup \mathscr{R}_{1}$

(4) $\mathscr{R}_{1} \cap \mathscr{R}_{2} \cong \mathscr{R}_{2} \cap \mathscr{R}_{1}$

(5) $\mathscr{R}_{1} \cup\left(\mathscr{R}_{2} \cap \mathscr{R}_{3}\right) \cong\left(\mathscr{R}_{1} \cup \mathscr{R}_{2}\right) \cap\left(\mathscr{R}_{1} \cup \mathscr{R}_{2}\right)$

(6) The projections $p_{1}: V\left(\mathscr{R}_{1} \cup \mathscr{R}_{2}\right) \longrightarrow\left(\mathscr{R}_{1}\right)$ and $p_{2}: V\left(\mathscr{R}_{1} \cup \mathscr{R}_{2}\right) \longrightarrow V\left(\mathscr{R}_{2}\right)$ may not be weak homomorphisms

(7) The projections $p_{1}: V\left(\mathscr{R}_{1} \cap \mathscr{R}_{2}\right) \longrightarrow\left(\mathscr{R}_{1}\right)$ and $p_{2}: V\left(\mathscr{R}_{1} \cap \mathscr{R}_{2}\right) \longrightarrow V\left(\mathscr{R}_{2}\right)$ may not be weak homomorphisms

Remark 4. Let $\mathscr{R}_{1}=\left(\underline{\mathscr{R}}_{1}, \overline{\mathscr{R}}_{1}\right)$ and $\mathscr{R}_{2}=\left(\underline{\mathscr{R}}_{2}, \overline{\mathscr{R}}_{2}\right)$ be two rough hypergraphs, then

(1) $\left[\mathscr{R}_{1} \cup \mathscr{R}_{2}\right]_{2} \cong\left[\mathscr{R}_{1}\right]_{2} \cup\left[\mathscr{R}_{2}\right]_{2}$

(2) $r\left(\mathscr{R}_{1} \cup \mathscr{R}_{2}\right)=\max \left\{r\left(\mathscr{R}_{1}\right), r\left(\mathscr{R}_{2}\right)\right.$, $\left.\left(r\left(\underline{\mathscr{R}}_{1}\right)+r\left(\overline{\mathscr{R}}_{2}\right) / 2\right),\left(r\left(\underline{\mathscr{R}}_{2}\right)+r\left(\overline{\mathscr{R}}_{1}\right) / 2\right)\right\}$

(3) $s\left(\mathscr{R}_{1} \cup \mathscr{R}_{2}\right)=\min \left\{s\left(\mathscr{R}_{1}\right), s\left(\mathscr{R}_{2}\right),\left(s\left(\underline{\mathscr{R}}_{1}\right)+\right.\right.$ $\left.\left.s\left(\overline{\mathscr{R}}_{2}\right) / 2\right),\left(s\left(\underline{\mathscr{R}}_{2}\right)+s\left(\overline{\mathscr{R}}_{1}\right) / 2\right)\right\}$

(4) $\left(\mathscr{R}_{1} \cup \mathscr{R}_{2}\right)^{*} \cong \mathscr{R}_{1}^{*} \cup \mathscr{R}_{2}^{*}$
3.5. Strong Product. In this subsection, we introduce the concept of a strong product using the Cartesian and square product of rough hypergraphs. We illustrate the notions of associativity, commutativity, distributivity, 2-section, distance, rank, and antirank properties of the strong product of rough hypergraphs.

Definition 34. Let $\mathscr{R}_{1}=\left(\underline{\mathscr{R}}_{1}, \overline{\mathscr{R}}_{1}\right)$ and $\mathscr{R}_{2}=\left(\underline{\mathscr{R}}_{2}, \overline{\mathscr{R}}_{2}\right)$ be two rough hypergraphs. The strong product of $\mathscr{R}_{1}$ and $\mathscr{R}_{2}$ is a rough hypergraph $\mathscr{R}_{1} \otimes \mathscr{R}_{2}=\left(\underline{\mathscr{R}}_{1} \otimes \mathscr{R}_{2}, \overline{\mathscr{R}}_{1} \otimes \overline{\mathscr{R}}_{2}\right)$, where $\underline{\mathscr{R}}_{1} \otimes \underline{\mathscr{R}}_{2}=\left(\underline{\mathscr{R}}_{1} \square \underline{\mathscr{R}}_{2}\right) \cup\left(\underline{\mathscr{R}}_{1} \times \underline{\mathscr{R}}_{2}\right)=\left(\underline{\varphi} A_{1} \times \underline{\varphi} A_{2},\left(\psi \underline{D}_{1}\right.\right.$ $\left.\left.\square \psi D_{2}\right) \cup\left(\psi D_{1} \times \psi D_{2}\right)\right)$ and $\overline{\mathscr{R}}_{1} \cup \overline{\mathscr{R}}_{2}=\left(\overline{\mathscr{R}}_{1} \square \overline{\mathscr{R}}_{2}\right) \cup$ $\left(\overline{\mathscr{R}}_{1} \times \overline{\mathscr{R}}_{2}\right)=\left(\bar{\varphi} A_{1} \times \bar{\varphi} A_{2},\left(\bar{\psi} D_{1} \square \bar{\psi} D_{2}\right) \cup\left(\bar{\psi} D_{1} \times \bar{\psi} D_{2}\right)\right)$. In other words, the strong product of $\mathscr{R}_{1}$ and $\mathscr{R}_{2}$ is the union of Cartesian product and square product of rough hypergraphs $\mathscr{R}_{1}$ and $\mathscr{R}_{2}$.

In short, $\underline{\mathscr{R}}_{1} \otimes \mathscr{R}_{2}$ and $\overline{\mathscr{R}}_{1} \otimes \overline{\mathscr{R}}_{2}$ are the strong products of lower approximate hypergraphs $\underline{\mathscr{R}}_{1}, \underline{\mathscr{R}}_{2}$ and upper approximate hypergraphs $\overline{\mathscr{R}}_{1}, \overline{\mathscr{R}}_{2}$, respectively. Just like the strong product of hypergraphs, the strong product of rough hypergraphs is associative, right distributive with respect to the disjoint union, commutative, and a unit $\mathcal{U}$ as a trivial hypergraph with a single vertex such that $\underline{\mathcal{U}}=\overline{\mathscr{U}}$. That is, for any rough hypergraphs $\mathscr{R}, \mathscr{R}_{1}, \mathscr{R}_{2}$, and $\mathscr{R}_{3}$, the following properties hold:

(1) $\mathscr{R}_{1} \otimes\left(\mathscr{R}_{2} \otimes \mathscr{R}_{3}\right) \cong\left(\mathscr{R}_{1} \otimes \mathscr{R}_{2}\right) \otimes \mathscr{R}_{3}$

(2) $\mathscr{R}_{1} \otimes \mathscr{R}_{2} \cong \mathscr{R}_{2} \otimes \mathscr{R}_{1}$

(3) $\mathscr{R}_{1} \otimes\left(\mathscr{R}_{2} \cup \mathscr{R}_{3}\right) \cong\left(\mathscr{R}_{1} \otimes \mathscr{R}_{2}\right) \cup\left(\mathscr{R}_{1} \otimes \mathscr{R}_{2}\right)$

(4) $\mathscr{R} \otimes \mathscr{U} \cong \mathscr{R}$, where $\mathcal{U}$ is a single vertex hypergraph without loops

(5) The projections $p_{1}: V\left(\mathscr{R}_{1} \otimes \mathscr{R}_{2}\right) \longrightarrow\left(\mathscr{R}_{1}\right)$ and $p_{2}: V\left(\mathscr{R}_{1} \otimes \mathscr{R}_{2}\right) \longrightarrow V\left(\mathscr{R}_{2}\right)$ may not be weak homomorphisms

(6) $\left[\mathscr{R}_{1} \otimes \mathscr{R}_{2}\right]_{2} \cong\left[\mathscr{R}_{1}\right]_{2} \otimes\left[\mathscr{R}_{2}\right]_{2}$

(7) $r\left(\mathscr{R}_{1} \otimes \mathscr{R}_{2}\right)=\quad \max \left\{r\left(\mathscr{R}_{1}\right), r\left(\mathscr{R}_{2}\right),\left(r\left(\underline{\mathscr{R}}_{1}\right)+\right.\right.$ $\left.\left.r\left(\overline{\mathscr{R}}_{2}\right) / 2\right),\left(r\left(\underline{\mathscr{R}}_{2}\right)+r\left(\overline{\mathscr{R}}_{1}\right) / 2\right)\right\}$

(8) $s\left(\mathscr{R}_{1} \otimes \mathscr{R}_{2}\right)=\min \left\{s\left(\mathscr{R}_{1}\right), s\left(\mathscr{R}_{2}\right)\right.$, $\left.\left(s\left(\underline{\mathscr{R}}_{1}\right)+s\left(\overline{\mathscr{R}}_{2}\right) / 2\right),\left(s\left(\underline{\mathscr{R}}_{2}\right)+s\left(\overline{\mathscr{R}}_{1}\right) / 2\right)\right\}$

Theorem 12. Let $\mathscr{R}_{1}$ and $\mathscr{R}_{2}$ be two rough hypergraphs on $Q_{1}$ and $Q_{2}$; then, for any $k_{1}, g_{1} \in Q_{1}$ and $k_{2}, g_{2} \in Q_{2}$,

$$
\begin{aligned}
& d_{\mathscr{R}_{1} \otimes \mathscr{R}_{2}}\left(\left(k_{1}, k_{2}\right),\left(g_{1}, g_{2}\right)\right) \\
& \quad=\left\{d_{\mathscr{R}_{1}}\left(k_{1}, g_{1}\right), d_{\mathscr{R}_{2}}\left(k_{2}, g_{2}\right), \frac{d_{\mathscr{R}_{1}}\left(k_{1}, g_{1}\right)+d_{\overline{\mathscr{R}}_{2}}\left(k_{2}, g_{2}\right)}{2}, \frac{d_{\overline{\mathscr{R}}_{1}}\left(k_{1}, g_{1}\right)+d_{\mathscr{\mathscr { R }}_{2}}\left(k_{2}, g_{2}\right)}{2}\right\} .
\end{aligned}
$$

Proof. The proof of this theorem is a direct consequence of Proposition 5.4 of [5], Lemma 2, and the result $\left[\mathscr{R}_{1} \otimes \mathscr{R}_{2}\right]_{2} \cong\left[\mathscr{R}_{1}\right]_{2} \otimes\left[\mathscr{R}_{2}\right]_{2}$. Thus, for any two rough hypergraphs $\mathscr{R}_{1}$ and $\mathscr{R}_{2}$, 


$$
\begin{aligned}
d_{\underline{\mathscr{R}}_{1} \otimes \underline{\mathscr{R}}_{2}}\left(\left(k_{1}, k_{2}\right),\left(g_{1}, g_{2}\right)\right) & =d_{\left[\underline{\mathscr{R}}_{1} \otimes \underline{\mathscr{R}}_{2}\right]_{2}}\left(\left(k_{1}, k_{2}\right),\left(g_{1}, g_{2}\right)\right) \\
& =d_{\left[\mathscr{R}_{1}\right]_{2} \otimes\left[\mathscr{R}_{2}\right]_{2}}\left(\left(k_{1}, k_{2}\right),\left(g_{1}, g_{2}\right)\right) \\
& =\max \left\{d_{\left[\underline{\mathscr{R}}_{1}\right]_{2}}\left(k_{1}, g_{1}\right), d_{\left[\mathscr{R}_{2}\right]_{2}}\left(k_{2}, g_{2}\right)\right\} \\
& \Rightarrow d_{\underline{\mathscr{R}}_{1} \otimes \underline{\mathscr{R}}_{2}}\left(\left(k_{1}, k_{2}\right),\left(g_{1}, g_{2}\right)\right) \\
& =\max \left\{d_{\underline{\mathscr{R}}_{1}}\left(k_{1}, g_{1}\right), d_{\underline{\mathscr{R}}_{2}}\left(k_{2}, g_{2}\right)\right\} .
\end{aligned}
$$

Similarly, $d_{\mathscr{R}_{1} \otimes \mathscr{R}_{2}}\left(\left(k_{1}, k_{2}\right),\left(g_{1}, g_{2}\right)\right)=\max \left\{d_{\mathscr{R}_{1}}\left(k_{1}, g_{1}\right)\right.$, $\left.d_{\mathscr{R}_{2}}\left(k_{2}, g_{2}\right)\right\}$, and the result follows.

3.6. Normal Product. In this subsection, we introduce the concept of a normal product using the Cartesian and direct product of rough hypergraphs. We elaborate on the notions of associativity, commutativity, distributivity, 2-section, distance, rank, and antirank properties of the normal product of rough hypergraphs.

Definition 35. Let $\mathscr{R}_{1}=\left(\underline{\mathscr{R}}_{1}, \overline{\mathscr{R}}_{1}\right)$ and $\mathscr{R}_{2}=\left(\underline{\mathscr{R}}_{2}, \overline{\mathscr{R}}_{2}\right)$ be two rough hypergraphs. The strong product of $\mathscr{R}_{1}$ and $\mathscr{R}_{2}$ is a rough hypergraph $\mathscr{R}_{1} \bar{\nabla} \mathscr{R}_{2}=\left(\underline{\mathscr{R}}_{1} \breve{\otimes}_{\mathscr{R}_{2}}, \overline{\mathscr{R}}_{1} \bar{\nabla} \overline{\mathscr{R}}_{2}\right)$, where $\underline{\mathscr{R}}_{1} \underline{\bar{Q}}_{\mathscr{R}_{2}}=\left(\underline{\mathscr{R}}_{1} \square \underline{\mathscr{R}}_{2}\right) \cup\left(\underline{\mathscr{R}}_{1} \times \underline{\mathscr{R}}_{2}\right)=\left(\varphi A_{1} \times \varphi A_{2},\left(\psi D_{1} \square\right.\right.$ $\left.\left.\psi D_{2}\right) \cup\left(\psi D_{1} \times \quad \psi D_{2}\right)\right) \quad$ and $\quad \overline{\mathscr{R}}_{1} \bar{\otimes} \overline{\mathscr{R}}_{2}=\left(\overline{\overline{\mathscr{R}}}_{1} \square \quad \overline{\mathscr{R}}_{2}\right) \cup$ $\left(\overline{\mathscr{R}}_{1} \overline{\times} \overline{\mathscr{R}}_{2}\right)=\left(\bar{\varphi} A_{1} \times \bar{\varphi} A_{2},\left(\bar{\psi} D_{1} \square \bar{\psi} D_{2}\right) \cup\left(\bar{\psi} D_{1} \bar{\psi} \bar{\psi} D_{2}\right)\right)$. In other words, the strong product of $\mathscr{R}_{1}$ and $\mathscr{R}_{2}$ is the union of Cartesian product and direct product of rough hypergraphs $\mathscr{R}_{1}$ and $\mathscr{R}_{2}$.

In short, $\mathscr{R}_{1} \varangle \mathscr{R}_{2}$ and $\overline{\mathscr{R}}_{1} \bar{\otimes} \overline{\mathscr{R}}_{2}$ are the strong products of lower approximate hypergraphs $\underline{\mathscr{R}}_{1}, \underline{R}_{2}$ and upper approximate hypergraphs $\overline{\mathscr{R}}_{1}, \overline{\mathscr{R}}_{2}$, respectively. Just like the strong product of hypergraphs, the strong product of rough hypergraphs is associative, right distributive with respect to the disjoint union, commutative, and a unit $\mathscr{U}$ as a trivial hypergraph with a single vertex such that $\underline{\mathcal{U}}=\overline{\mathscr{U}}$. That is, for any rough hypergraphs $\mathscr{R}, \mathscr{R}_{1}, \mathscr{R}_{2}$, and $\mathscr{R}_{3}$, the following properties hold:

(1) $\mathscr{R}_{1} \breve{\otimes}\left(\mathscr{R}_{2} \breve{\mathrm{Q}}_{3}\right) \cong\left(\mathscr{R}_{1} \overline{\mathrm{\nabla}} \mathscr{R}_{2}\right) \overline{\mathrm{\otimes}} \mathscr{R}_{3}$

(2) $\mathscr{R}_{1} \widetilde{\nabla} \mathscr{R}_{2} \cong \mathscr{R}_{2} \widetilde{\nabla} \mathscr{R}_{1}$

(3) $\mathscr{R}_{1} \widetilde{\otimes}\left(\mathscr{R}_{2} \cup \mathscr{R}_{3}\right) \cong\left(\mathscr{R}_{1} \widetilde{\otimes} \mathscr{R}_{2}\right) \cup\left(\mathscr{R}_{1} \breve{\otimes}_{2}\right)$

(4) $\mathscr{R} \bowtie \mathcal{U} \cong \mathscr{R}$, where $\mathscr{U}$ is a single vertex hypergraph without loops

(5) The projections $p_{1}: V\left(\mathscr{R}_{1} \breve{\otimes}_{2}\right) \longrightarrow\left(\mathscr{R}_{1}\right)$ and $p_{2}: V\left(\mathscr{R}_{1} \otimes \mathscr{R}_{2}\right) \longrightarrow V\left(\mathscr{R}_{2}\right)$ may not be weak homomorphisms

(6) $\left[\mathscr{R}_{1} \breve{\otimes} \mathscr{R}_{2}\right]_{2} \cong\left[\mathscr{R}_{1}\right]_{2} \otimes\left[\mathscr{R}_{2}\right]_{2}$

(7) $r\left(\mathscr{R}_{1} \breve{\otimes}_{2} \mathscr{R}_{2}\right)=\max \left\{r\left(\mathscr{R}_{1}\right)\right.$, $\left.r\left(\mathscr{R}_{2}\right),\left(r\left(\underline{\mathscr{R}}_{1}\right)+r\left(\overline{\mathscr{R}}_{2}\right) / 2\right),\left(r\left(\underline{\mathscr{R}}_{2}\right)+r\left(\overline{\mathscr{R}}_{1}\right) / 2\right)\right\}$

(8) $s\left(\mathscr{R}_{1} \breve{\nabla}_{2}\right)=\min \left\{s\left(\mathscr{R}_{1}\right), s\left(\mathscr{R}_{2}\right)\right.$, $\left.\left(s\left(\underline{\mathscr{R}}_{1}\right)+s\left(\overline{\mathscr{R}}_{2}\right) / 2\right),\left(s\left(\underline{\mathscr{R}}_{2}\right)+s\left(\overline{\mathscr{R}}_{1}\right) / 2\right)\right\}$

Theorem 13. Let $\mathscr{R}_{1}$ and $\mathscr{R}_{2}$ be two rough hypergraphs on $Q_{1}$ and $Q_{2}$, then for any $k_{1}, g_{1} \in Q_{1}$ and $k_{2}, g_{2} \in Q_{2}$,

$$
\begin{aligned}
& d_{\mathscr{R}_{1} \overleftarrow{\otimes} \mathscr{R}_{2}}\left(\left(k_{1}, k_{2}\right),\left(g_{1}, g_{2}\right)\right) \\
& \quad=\left\{d_{\mathscr{R}_{1}}\left(k_{1}, g_{1}\right), d_{\mathscr{R}_{2}}\left(k_{2}, g_{2}\right),\right. \\
& \left.\quad \frac{d_{\mathscr{R}_{1}}\left(k_{1}, g_{1}\right)+d_{\overline{\mathscr{R}}_{2}}\left(k_{2}, g_{2}\right)}{2}, \frac{d_{\overline{\mathscr{R}}_{1}}\left(k_{1}, g_{1}\right)+d_{\mathscr{R}_{2}}\left(k_{2}, g_{2}\right)}{2}\right\} .
\end{aligned}
$$

Proof. The proof of this theorem is a direct consequence of Proposition 5.4 of [5], Lemma 2 and the result $\left[\mathscr{R}_{1} \otimes \mathscr{R}_{2}\right]_{2} \cong\left[\mathscr{R}_{1}\right]_{2} \otimes\left[\mathscr{R}_{2}\right]_{2}$. Thus, for any two rough hypergraphs $\mathscr{R}_{1}$ and $\mathscr{R}_{2}$.

3.7. Lexicographic Product and Costrong Product. In this subsection, we describe the properties of the lexicographic product and costrong product of rough hypergraphs.

Definition 36. Let $\mathscr{R}_{1}=\left(\underline{\mathscr{R}}_{1}, \overline{\mathscr{R}}_{1}\right)$ and $\mathscr{R}_{2}=\left(\underline{\mathscr{R}}_{2}, \overline{\mathscr{R}}_{2}\right)$ be two rough hypergraphs. The lexicographic product ${ }^{\circ}$ of $\mathscr{R}_{1}$ and $\mathscr{R}_{2}$ is a rough hypergraph $\mathscr{R}_{1} \circ \mathscr{R}_{2}=\left(\underline{\mathscr{R}}_{1} \circ \underline{\mathscr{R}}_{2}, \overline{\mathscr{R}}_{1} \circ \overline{\mathscr{R}}_{2}\right)$ which is defined as

(1) $\underline{\mathscr{R}}_{1} \circ \underline{\mathscr{R}}_{2}=\left(\underline{\varphi} \mathrm{A}_{1} \times \underline{\varphi} \mathrm{A}_{2}, \underline{\psi} \mathrm{D}_{1}^{\circ} \underline{\psi} \mathrm{D}_{2}\right)$

(a) $\varphi A_{1} \times \varphi A_{2}=\left\{\left(k_{1}, k_{2}\right) \mid k_{1} \in \varphi A_{1}, k_{2} \in \varphi A_{2}\right\}$

(b) $\psi D_{1}^{\circ} \psi \mathrm{D}_{2}=\left\{\underline{\mathrm{E}} \mid \mathrm{p}_{1}(\underline{\mathrm{E}}) \in \psi \mathrm{D}_{1}, \quad p_{2}(\underline{E}) \subseteq \underline{E}_{2} \in\right.$ $\left.\bar{\psi} D_{2}\right\} \bar{\cup}\left\{\left\{k_{1}\right\} \times \underline{E}_{2} \mid k_{1} \in \psi \bar{D}_{1}, \underline{E}_{2} \in \psi \underline{D}_{2}\right\}$

(2) $\overline{\mathscr{R}}_{1} \circ \overline{\mathscr{R}}_{2}=\left(\bar{\varphi} \mathrm{A}_{1} \times \bar{\varphi} \mathrm{A}_{2}, \bar{\psi} \mathrm{D}_{1}^{\circ} \bar{\psi} \mathrm{D}_{2}\right)$

(a) $\bar{\varphi} A_{1} \times \bar{\varphi} A_{2}=\left\{\left(k_{1}, k_{2}\right) \mid k_{1} \in \bar{\varphi} A_{1}, k_{2} \in \bar{\varphi} A_{2}\right\}$

(b) $\bar{\psi} D_{1}^{\circ} \bar{\psi} D_{2}=\left\{\bar{E} \mid p_{1}(\bar{E}) \in \bar{\psi} D_{1}, p_{2}(\bar{E}) \subseteq \bar{E}_{2} \in\right.$ $\left.\bar{\psi} D_{2}\right\} \cup\left\{\left\{k_{1}\right\} \times \bar{E}_{2} \mid k_{1} \in \bar{\psi} D_{1}, \bar{E}_{2} \in \bar{\psi} D_{2}\right\}$

In short, $\underline{\mathscr{R}}_{1} \circ \underline{\mathscr{R}}_{2}$ and $\overline{\mathscr{R}}_{1} \circ \overline{\mathscr{R}}_{2}$ are the lexicographic products of lower approximate hypergraphs $\underline{\mathscr{R}}_{1}, \underline{\mathscr{R}}_{2}$ and upper approximate hypergraphs $\overline{\mathscr{R}}_{1}, \overline{\mathscr{R}}_{2}$, respectively. Just like the lexicographic product of hypergraphs, the lexicographic product of rough hypergraphs is associative, right distributive with respect to the disjoint union, noncommutative, and a unit $\mathscr{U}$ (left identity) as a trivial hypergraph with a single vertex such that $\underline{\mathcal{U}}=\overline{\mathscr{U}}$. That is, for any rough hypergraphs $\mathscr{R}, \mathscr{R}_{1}, \mathscr{R}_{2}$, and $\mathscr{R}_{3}$, the following properties hold:

(1) $\mathscr{R}_{1} \circ\left(\mathscr{R}_{2} \circ \mathscr{R}_{3}\right) \cong\left(\mathscr{R}_{1} \circ \mathscr{R}_{2}\right) \circ \mathscr{R}_{3}$

(2) $\mathscr{R}_{1} \circ \mathscr{R}_{2} \cong \mathscr{R}_{2} \circ \mathscr{R}_{1}$

(3) $\mathscr{R}_{1} \circ\left(\mathscr{R}_{2} \cup \mathscr{R}_{3}\right) \cong\left(\mathscr{R}_{1} \circ \mathscr{R}_{2}\right) \cup\left(\mathscr{R}_{1} \circ \mathscr{R}_{2}\right)$

(4) $\mathcal{U} \mathscr{R} \cong \mathscr{R}$, where $\mathcal{U}$ is a single vertex hypergraph without loops

(5) $\mathscr{R} \circ \mathscr{U} \cong \mathscr{R}$

(6) The projections $p_{1}: V\left(\mathscr{R}_{1} \circ \mathscr{R}_{2}\right) \longrightarrow\left(\mathscr{R}_{1}\right)$ and $p_{2}: V\left(\mathscr{R}_{1} \circ \mathscr{R}_{2}\right) \longrightarrow \mathrm{V}\left(\mathscr{R}_{2}\right)$ may not be weak homomorphisms

(7) $\left[\mathscr{R}_{1} \circ \mathscr{R}_{2}\right]_{2} \cong\left[\mathscr{R}_{1}\right]_{2}^{\circ}\left[\mathscr{R}_{2}\right]_{2}$

(8) $r\left(\mathscr{R}_{1} \circ \mathscr{R}_{2}\right)=\max \left\{\mathrm{r}\left(\mathscr{R}_{1}\right)\right.$, $\left.r\left(\mathscr{R}_{2}\right),\left(r\left(\underline{\mathscr{R}}_{1}\right)+r\left(\overline{\mathscr{R}}_{2}\right) / 2\right),\left(r\left(\underline{\mathscr{R}}_{2}\right)+r\left(\overline{\mathscr{R}}_{1}\right) / 2\right)\right\}$ 
(9) $s\left(\mathscr{R}_{1} \circ \mathscr{R}_{2}\right)=\min \left\{s\left(\mathscr{R}_{1}\right)\right.$, $\left.s\left(\mathscr{R}_{2}\right),\left(s\left(\underline{\mathscr{R}}_{1}\right)+s\left(\overline{\mathscr{R}}_{2}\right) / 2\right),\left(s\left(\underline{\mathscr{R}}_{2}\right)+s\left(\overline{\mathscr{R}}_{1}\right) / 2\right)\right\}$
Theorem 14. Let $\mathscr{R}_{1}$ and $\mathscr{R}_{2}$ be two rough hypergraphs on $Q_{1}$ and $Q_{2}$; then, for any $k_{1}, g_{1} \in Q_{1}$ and $k_{2}, g_{2} \in Q_{2}$,

$$
\begin{aligned}
& d_{\underline{\mathscr{R}}_{1} \circ \mathscr{\mathscr { R }}_{2}}\left(\left(k_{1}, k_{2}\right),\left(g_{1}, g_{2}\right)\right)= \begin{cases}d_{\mathscr{R}_{1}}\left(k_{1}, g_{1}\right), & \text { if } k_{1} \neq g_{1}, \\
d_{\mathscr{R}_{2}}\left(k_{2}, g_{2}\right), & \text { if } k_{1}=g_{1}, \operatorname{deg}\left(k_{1}\right)=0, \\
\min \left\{d_{\mathscr{R}_{2}}\left(k_{2}, g_{2}\right), 2\right\}, & \text { if } k_{1}=g_{1}, \operatorname{deg}\left(k_{1}\right) \neq 0,\end{cases} \\
& d_{\overline{\mathscr{R}}_{1} \circ \overline{\mathscr{R}}_{2}}\left(\left(k_{1}, k_{2}\right),\left(g_{1}, g_{2}\right)\right)= \begin{cases}d_{\overline{\mathscr{R}}_{1}}\left(k_{1}, g_{1}\right), & \text { if } k_{1} \neq g_{1}, \\
d_{\overline{\mathscr{R}}_{2}}\left(k_{2}, g_{2}\right), & \text { if } k_{1}=g_{1}, \operatorname{deg}\left(k_{1}\right)=0, \\
\min \left\{d_{\overline{\mathscr{R}_{2}}}\left(k_{2}, g_{2}\right), 2\right\}, & \text { if } k_{1}=g_{1}, \operatorname{deg}\left(k_{1}\right) \neq 0 .\end{cases}
\end{aligned}
$$

Proof. The proof of this theorem is a direct consequence of Proposition 5.4 of [5], Lemma 2, and the result
$\left[\mathscr{R}_{1} \otimes \mathscr{R}_{2}\right]_{2} \cong\left[\mathscr{R}_{1}\right]_{2} \otimes\left[\mathscr{R}_{2}\right]_{2}$. Thus, for any two rough hypergraphs $\mathscr{R}_{1}$ and $\mathscr{R}_{2}$,

$$
\begin{aligned}
& d_{\underline{\mathscr{R}}_{1} \circ \mathscr{R}_{2}}\left(\left(k_{1}, k_{2}\right),\left(g_{1}, g_{2}\right)\right)=d_{\left[\underline{\mathscr{R}}_{1}^{\circ} \stackrel{\mathscr{R}}{2}_{2}\right]_{2}}\left(\left(k_{1}, k_{2}\right),\left(g_{1}, g_{2}\right)\right) \\
& =d_{\left[\mathscr{R}_{1}\right]_{2}{ }^{\circ}\left[\mathscr{R}_{2}\right]_{2}}\left(\left(k_{1}, k_{2}\right),\left(g_{1}, g_{2}\right)\right) \\
& = \begin{cases}d_{\left[\mathscr{R}_{1}\right]_{2}}\left(k_{1}, g_{1}\right), & \text { if } k_{1} \neq g_{1}, \\
d_{\mathscr{R}_{2}}\left(k_{2}, g_{2}\right), & \text { if } k_{1}=g_{1}, \operatorname{deg}\left(k_{1}\right)=0, \\
\min \left\{d_{\mathscr{R}_{2}}\left(k_{2}, g_{2}\right), 2\right\} & \text { if } k_{1}=g_{1}, \operatorname{deg}\left(k_{1}\right) \neq 0,\end{cases} \\
& \Rightarrow d_{\underline{\mathscr{R}}_{1}{ }^{\circ} \mathscr{\mathscr { R }}_{2}}\left(\left(k_{1}, k_{2}\right),\left(g_{1}, g_{2}\right)\right)= \begin{cases}d_{\mathscr{R}_{1}}\left(k_{1}, g_{1}\right), & \text { if } k_{1} \neq g_{1}, \\
d_{\mathscr{R}_{2}}\left(k_{2}, g_{2}\right), & \text { if } k_{1}=g_{1}, \operatorname{deg}\left(k_{1}\right)=0, \\
\min \left\{d_{\mathscr{R}_{2}}\left(k_{2}, g_{2}\right), 2\right\}, & \text { if } k_{1}=g_{1}, \operatorname{deg}\left(k_{1}\right) \neq 0 .\end{cases}
\end{aligned}
$$

Similarly for upper approximate hypergraphs, the result follows.

Definition 37. Let $\mathscr{R}_{1}=\left(\underline{\mathscr{R}}_{1}, \overline{\mathscr{R}}_{1}\right)$ and $\mathscr{R}_{2}=\left(\underline{\mathscr{R}}_{2}, \overline{\mathscr{R}}_{2}\right)$ be two rough hypergraphs. The costrong product $\star$ of $\mathscr{R}_{1}$ and $\mathscr{R}_{2}$ is a rough hypergraph $\mathscr{R}_{1} \star \mathscr{R}_{2}=\left(\underline{\mathscr{R}}_{1} \star \mathscr{R}_{2}, \overline{\mathscr{R}}_{1} \star \overline{\mathscr{R}}_{2}\right)$ which is defined as $\mathscr{R}_{1} \star \mathscr{R}_{2}=\left(\mathscr{R}_{1} \circ \mathscr{R}_{2}\right) \cup\left(\mathscr{R}_{2} \circ \mathscr{R}_{1}\right)$.

In short, $\underline{\mathscr{R}}_{1} \circ \mathscr{\mathscr { R }}_{2}$ and $\overline{\mathscr{R}}_{1} \circ \overline{\mathscr{R}}_{2}$ are the costrong products of lower approximate hypergraphs $\mathscr{R}_{1}, \mathscr{R}_{2}$ and upper approximate hypergraphs $\overline{\mathscr{R}}_{1}, \overline{\mathscr{R}}_{2}$, respectively. Just like the costrong product of hypergraphs, the costrong product of rough hypergraphs is associative, right distributive with respect to the disjoint union, commutative, and a unit $\mathscr{U}$ (left identity) as a trivial hypergraph with a single vertex such that $\underline{u}=\bar{u}$.

Remark 5. Let $\mathscr{R}_{1}$ and $\mathscr{R}_{2}$ be two rough hypergraphs, then

(1) $\left[\mathscr{R}_{1} \star \mathscr{R}_{2}\right]_{2}=\left[\mathscr{R}_{1}\right]_{2} \star\left[\mathscr{R}_{2}\right]_{2}$

(2) $r\left(\mathscr{R}_{1} \star \mathscr{R}_{2}\right)=\max \left\{r\left(\mathscr{R}_{1}\right)\right.$, $\left.r\left(\mathscr{R}_{2}\right),\left(r\left(\underline{\mathscr{R}}_{1}\right)+r\left(\mathscr{\mathscr { R }}_{2}\right) / 2\right),\left(r\left(\underline{\mathscr{R}}_{2}\right)+r\left(\overline{\mathscr{R}}_{1}\right) / 2\right)\right\}$

(3) $s\left(\mathscr{R}_{1} \star \mathscr{R}_{2}\right)=\min \left\{s\left(\mathscr{R}_{1}\right), s\left(\mathscr{R}_{2}\right),\left(s\left(\mathscr{R}_{1}\right)+\right.\right.$ $\left.\left.s\left(\overline{\mathscr{R}}_{2}\right) / 2\right),\left(s\left(\underline{\mathscr{R}}_{2}\right)+s\left(\overline{\mathscr{R}}_{1}\right) / 2\right)\right\}$
3.8. Limitations of the Proposed Study. Apart from all the benefits, rough hypergraphs also have some shortcomings and disadvantages. Rough sets and hypergraphs are both complex mathematical structures and are not simple to apply for the given information. The computation of rough relations using power sets is a lengthy and tricky task. There are a lot of complicated calculations which make it difficult to study hypergraphical structures using rough sets. The calculation complexity not only increases time consumption but also increases the probability of errors.

\section{Conclusions and Future Directions}

Rough models combined with other algebraic structures retain the property to study uncertain and vague information using approximation techniques. To discuss approximate relations among more than two objects, rough graphs cannot give error-free results. In this research paper, the notion of a rough set was applied to hypergraphs to introduce the novel concept of rough hypergraphs. Certain important properties of isomorphism, conformality, linearity, duality, associativity, commutativity, distributivity, Helly property, and intersecting families of rough hypergraphs are illustrated in detail. The formulae of distance 
function, 2-section, L2-section, covering, coloring, rank, and antirank of certain products of rough hypergraphs are established in terms of corresponding rough hypergraphs. This work can further be extended to (1) Dombi fuzzy rough hypergraphs, (2) bipolar fuzzy rough hypergraphs, and (3) picture fuzzy rough hypergraphs.

\section{Data Availability}

No data were used to support this study.

\section{Ethical Approval}

This article does not contain any studies with human participants or animals performed by the author.

\section{Conflicts of Interest}

The author declares she has no conflicts of interest regarding the publication of this research article.

\section{References}

[1] C. Berge, Hypergraphs: Combinatorics of Finite Sets, Vol. 45, North-Holland, Amsterdam, Netherlands, 1989.

[2] A. Bretto, Y. Silvestre, and T. Vallée, "Cartesian product of hypergraphs: properties and algorithms," Electronic Proceedings in Theoretical Computer Science, vol. 4, pp. 22-28, 2009.

[3] W. Dörfler, "On the direct product of hypergraphs," Ars Combinatoria, vol. 14, pp. 67-78, 1982.

[4] M. Sonntag, "Hamiltonicity of products of hypergraphs," Annals of Discrete Mathematics, vol. 51, pp. 329-332, 1992.

[5] R. Hammack, W. Imrich, and S. Klavžar, "Handbook of product graphs," Discrete Mathematics and its Applications, CRC Press, Boca Raton, FL, USA, 2nd edition, 2011.

[6] A. Kaufmann, Introduction la Thorie Des Sous-Ensembles Flous à L'Usage Des Ingnieurs (Fuzzy Sets Theory), Masson, Paris, France, 1973.

[7] L. A. Zadeh, "Fuzzy sets," Information and Control, vol. 8, no. 3, pp. 338-353, 1965.

[8] H. Lee-Kwang and K.-M. Lee, "Fuzzy hypergraph and fuzzy partition," IEEE Transactions on Systems, Man, and Cybernetics, vol. 25, no. 1, pp. 196-201, 1995.

[9] C. Radhamani and C. Radhika, "Isomorphism on fuzzy hypergraphs," IOSR Journal of Mathematics, vol. 2, no. 6, pp. 24-31, 2012.

[10] R. H. Goetschel Jr, "Introduction to fuzzy hypergraphs and Hebbian structures," Fuzzy Sets and Systems, vol. 76, no. 1, pp. 113-130, 1995.

[11] R. H. Goetschel Jr, "Fuzzy colorings of fuzzy hypergraphs," Fuzzy Sets and Systems, vol. 94, no. 2, pp. 185-204, 1998.

[12] R. H. Goetschel Jr, W. L. Craine, and W. Voxman, "Fuzzy transversals of fuzzy hypergraphs," Fuzzy Sets and Systems, vol. 84, no. 3, pp. 235-254, 1996.

[13] J. N. Mordeson and P. S. Nair, "Fuzzy graphs and hypergraphs," Studies in Fuzziness and Soft Computing, Springer, Berlin, Germany, 2nd edition, 1998.

[14] M. Akram, M. Sarwar, and R. A. Borzooei, "A novel decisionmaking approach based on hypergraphs in intuitionistic fuzzy environment," Journal of Intelligent and Fuzzy Systems, vol. 35, no. 2, pp. 1905-1922, 2018.
[15] R. Parvathi, S. Thilagavathi, and M. G. Karunambigai, "Intuitionistic fuzzy hypergraphs," Cybernetics and Information Technologies, vol. 9, no. 2, pp. 46-53, 2009.

[16] S. Samanta and M. Pal, "Bipolar fuzzy hypergraphs," International Journal of Fuzzy Logic Systems, vol. 2, no. 1, pp. 17-28, 2012.

[17] M. Akram and A. Luqman, "Fuzzy hypergraphs and related extensions," Studies in Fuzziness and Soft Computing, vol. 390, 2020.

[18] M. Akram and M. Sarwar, "Novel applications of $m$-polar fuzzy hypergraphs," Journal of Intelligent and Fuzzy Systems, vol. 32, no. 3, pp. 2747-2762, 2017.

[19] M. Akram and M. Sarwar, "Transversals of $m$-polar fuzzy hypergraphs with applications," Journal of Intelligent and Fuzzy Systems, vol. 33, no. 1, pp. 351-364, 2017.

[20] M. Sarwar, M. Akram, and S. Shahzadi, "Bipolar fuzzy soft information applied to hypergraphs," Soft Computing, vol. 25, no. 5, pp. 3417-3439, 2021.

[21] Z. A. Pawlak, "Rough sets," International Journal of Computer \& Information Sciences, vol. 11, no. 5, pp. 341-356, 1982.

[22] Z. Pawlak, "Rough sets, rough relations and rough functions," Fundamenta Informaticae, vol. 27, no. 2-3, pp. 103-108, 1996.

[23] M. Sarwar, "Decision-making approaches based on color spectrum and D-TOPSIS method under rough environment," Computational and Applied Mathematics, vol. 39, no. 4, pp. 1-32, 2020

[24] F. Zafar and M. Akram, "A novel decision-making method based on rough fuzzy information," International Journal of Fuzzy Systems, vol. 20, no. 3, pp. 1000-1014, 2018.

[25] M. Akram, Shumaiza, and M. Arshad, "A new approach based on fuzzy rough digraphs for decision-making," Journal of Intelligent and Fuzzy Systems, vol. 35, no. 2, pp. 2105-2121, 2018.

[26] M. Akram and F. Zafar, "Hybrid soft computing models applied to graph theory," Studies in Fuzziness and Soft Computing, vol. 380, 2020.

[27] F. Feng, C. Li, B. Davvaz, and M. I. Ali, "Soft sets combined with fuzzy sets and rough sets: a tentative approach," Soft Computing, vol. 14, no. 9, pp. 899-911, 2010.

[28] D. Meng, X. Zhang, and K. Qin, "Soft rough fuzzy sets and soft fuzzy rough sets," Computers \& Mathematics with Applications, vol. 62, no. 12, pp. 4635-4645, 2011.

[29] D. Dubois and H. Prade, "Rough fuzzy sets and fuzzy rough sets," International Journal of General Systems, vol. 17, no. 2-3, pp. 191-209, 1990.

[30] A. Hamed, A. Sobhy, and H. Nassar, "Distributed approach for computing rough set approximations of big incomplete information systems," Information Sciences, vol. 547, pp. 427-449, 2021.

[31] V. L. Fotea, "The lower and upper approximations in a hypergroup," Information Sciences, vol. 178, no. 18, pp. 3605-3615, 2008.

[32] J. Luo, H. Fujita, Y. Yao, and K. Qin, “On modeling similarity and three-way decision under incomplete information in rough set theory," Knowledge-Based Systems, vol. 191, Article ID 105251, 2020.

[33] M. C. Nicoletti, J. Q. Uchôa, and M. T. Baptistini, "Rough relation properties," International Journal of Applied Mathematics and Computer Science, vol. 11, no. 3, pp. 621-635, 2001.

[34] B. Barman and S. Patra, "Variable precision rough set based unsupervised band selection technique for hyperspectral image classification," Knowledge-Based Systems, vol. 193, Article ID 105414, 2020. 
[35] J. Chen, J. Mi, and Y. Lin, "A graph approach for fuzzyrough feature selection," Fuzzy Sets and Systems, vol. 391, pp. 96-116, 2020.

[36] Y. Kusunoki, J. Baszczyski, M. Inuiguchi, and R. Sowiski, "Empirical risk minimization for dominance-based rough set approaches," Information Sciences, vol. 567. , 2021 In press.

[37] M. J. Benítez-Caballero, J. Medina, E. Ramírez-Poussa, and D. Śl $\square$ zak, "Rough-set-driven approach for attribute reduction in fuzzy formal concept analysis," Fuzzy Sets and Systems, vol. 391, pp. 117-138, 2020.

[38] J. Chen and J. Li, "An application of rough sets to graph theory," Information Sciences, vol. 201, pp. 114-127, 2012.

[39] T. Jin, Z. Yu, Y. Gao, S. Gao, X. Sun, and C. Li, "Robust ใ2-hypergraph and its applications," Information Sciences, vol. 501, pp. 708-723, 2019.

[40] B. Mathew, S. J. John, and H. Garg, "Vertex rough graphs," Complex \& Intelligent Systems, vol. 6, no. 2, pp. 347-353, 2020.

[41] G. Cattaneo, G. Chiaselotti, D. Ciucci, and T. Gentile, "On the connection of hypergraph theory with formal concept analysis and rough set theory," Information Sciences, vol. 330, pp. 342-357, 2016.

[42] A. Semenov and D. Shabanov, "On the weak chromatic number of random hypergraphs," Discrete Applied Mathematics, vol. 276, pp. 134-154, 2020.

[43] C. Châtel, F. Brucker, and P. Préa, "Binary set systems and totally balanced hypergraphs," Discrete Applied Mathematics, vol. 295, pp. 120-133, 2021.

[44] E. SanJuan, "Heyting algebras with Boolean operators for rough sets and information retrieval applications," Discrete Applied Mathematics, vol. 156, no. 6, pp. 967-983, 2008.

[45] K. Engel and T. D. Thu, "Boundary optimization for rough sets," Discrete Mathematics, vol. 341, no. 9, pp. 2465-2477, 2018.

[46] T. K. Maryati and B. Davvaz, "A novel connection between rough sets, hypergraphs and hypergroups," Discrete Mathematics, Algorithms and Applications, vol. 9, no. 4, Article ID 1750044, 2017.

[47] M. Palangetić, C. Cornelis, S. Greco, and R. Sowiski, "Fuzzy extensions of the dominance-based rough set approach," International Journal of Approximate Reasoning, vol. 129, pp. 1-19, 2021.

[48] Q. Jin, L. Li, Z. Ma, and B. Yao, "A note on the relationships between generalized rough sets and topologies," International Journal of Approximate Reasoning, vol. 130, pp. 292-296, 2021.

[49] S. Naz, S. Ashraf, and H. Rashmanlou, "Measurement of planarity in product bipolar fuzzy graphs," TWMS Journal of Applied and Engineering Mathematics, vol. 9, no. 2, pp. 339-350, 2019.

[50] M. Akram, G. Ali, and M. Shabir, "A hybrid decision-making framework using rough $\mathrm{mF}$ bipolar soft environment," Granular Computing, vol. 6, no. 3, pp. 539-555, 2021.

[51] M. Akram and G. Ali, "Hybrid models for decision-making based on rough Pythagorean fuzzy bipolar soft information," Granular Computing, vol. 5, no. 1, pp. 1-15, 2020.

[52] W. Imrich and I. Peterin, "Recognizing cartesian products in linear time," Discrete Mathematics, vol. 307, no. 3-5, pp. 472-483, 2007. 